UG-30/99

\title{
Nontriviality of Abelian gauged Nambu-Jona-Lasinio models in four dimensions
}

\author{
Manuel Reenders* \\ Institute for Theoretical Physics, \\ University of Groningen, 9747 AG Groningen, The Netherlands
}

(April 2000)

\begin{abstract}
We study a particular class of Abelian gauged Nambu-Jona-Lasinio models with global $U_{L}(N) \times U_{R}(N)$ symmetry, where $N$ is the number of fermion flavors. We show, by treating the gauge interaction in the ladder approximation and four-fermion interactions in the leading order of the $1 / N$ expansion, that the renormalization-group $\beta$ function of the $U(1)$ gauge coupling has ultraviolet stable fixed points for sufficiently large $N$. This implies the existence of a nontrivial continuum limit.
\end{abstract}

11.10.Hi, 11.15.Tk, 11.30.Qc, 11.30.Rd

Typeset using REVTEX

*Email address: m.reenders@phys.rug.nl 


\section{INTRODUCTION}

The absence of an interacting continuum limit or triviality of Abelian gauge theories in four dimensions such as QED is due to the screening of charged particles by their interactions with virtual fermion-antifermion pairs from the vacuum. Such charge screening is described by the vacuum polarization $\Pi$ (Fig. 1). The QED vacuum is not a perfect insulator and can be considered as a medium of dipoles representing the fermion loops in the vacuum polarization. Within perturbation theory, the electromagnetic charge or gauge coupling is screened completely in the continuum limit $(\Lambda \rightarrow \infty$, where $\Lambda$ is the ultraviolet cutoff).

This can be seen by considering the renormalization group (RG) transformation of QED [1], which relates the gauge coupling or fine-structure constant $\alpha_{\mu}$ in the infrared (IR) region to the bare gauge coupling $\alpha_{0}=\alpha_{\Lambda}$ in the ultraviolet (UV) region via

$$
\alpha_{\mu}=\mathcal{R}_{\alpha}\left[\mu / \Lambda, \alpha_{0}\right], \quad \mathcal{R}_{\alpha}\left[\mu / \Lambda, \alpha_{0}\right]=\frac{\alpha_{0}}{1+\Pi\left[\mu / \Lambda, \alpha_{0}\right]}
$$

In perturbation theory, the one-loop leading contribution to $\Pi$ is

$$
\Pi\left[\mu / \Lambda, \alpha_{0}\right]=\frac{2 N \alpha_{0}}{3 \pi} \ln \frac{\Lambda}{\mu}
$$

where $N$ is the number of fermion flavors. This logarithmic screening effect is sufficient to cause the complete screening of charge in the continuum limit [2,3].

From the RG point of view triviality is merely due to the absence of an UV stable fixed point or nontrivial root of the $\beta$ function

$$
\left.\beta_{\alpha}\left(\alpha_{0}\right) \equiv \frac{\partial \mathcal{R}_{\alpha}\left[w, \alpha_{0}\right]}{\partial w}\right|_{w=1}=\Lambda \frac{d \alpha_{0}}{d \Lambda}
$$

for the gauge coupling. Equation (2) gives rise to the $\beta$ function $\beta_{\alpha}(\alpha)=2 N \alpha^{2} / 3 \pi$, which only has an IR stable fixed point or trivial root: $\alpha=0\left(\beta_{\alpha}^{\prime}(0) \geq 0\right)$. A nontrivial or interacting theory only arises whenever $\beta_{\alpha}$ has a root which is an UV stable fixed point. In case of asymptotic free theories such as QCD the origin $\alpha=0$ is an UV stable fixed point.

In addition to its motivation only on the level of perturbation theory the above consideration is too naive. According to the RG methods of Wilson [4,5], one should consider the $\mathrm{RG}$ flow in the space of all coupling constants (respecting certain symmetries) or at least for those coupling constants which can be classified as relevant close to a particular critical manifold in coupling constant space. For a possible nontrivial continuum limit of QED, critical and hence nonperturbative dynamical effects are required.

Therefore, the discovery of dynamical chiral symmetry breaking ( $\chi \chi \mathrm{SB})$ in the strong coupling phase of QED $\left(\alpha_{0} \geq \alpha_{c}=\pi / 3\right)$ [6] and the existence of an UV stable fixed point in the quenched-ladder approximation [9 11] sheds new light on the nonperturbative nature of QED and triviality. Lattice simulations of so-called noncompact quenched QED have confirmed the existence of a continuous chiral phase transition [12 [14].

An important step was performed by Bardeen, Leung, and Love in Refs. [15, 16]. These authors realized that, in quenched-ladder QED, attractive four-fermion interactions described by a dimensionless four-fermion coupling $g_{0} \geq 0$ have a so-called scaling dimension 4 
instead of 6 at the critical gauge coupling $\alpha_{0}=\alpha_{c}$. Consequently, these operators mix with the gauge interaction which also has dimension 4 in four space-time dimensions; this means that QED is not a closed theory at the chiral phase transition. The scaling dimension of an operator is important in determining whether or not such an operator describes a long-range interaction. The model of Bardeen, Leung, and Love is referred to as the gauged NambuJona-Lasinio (GNJL) model, and the mechanism of dynamical chiral symmetry breaking proposed by them should be regarded in the context of the RG methods of Wilson.

Wilson pointed out that a nontrivial renormalizable model can only be formulated if the RG transformation exhibits UV stable fixed points. UV stable fixed points are "singular" points of the RG transformation at which the model becomes scale (conformal) invariant. Natural candidates for UV stable fixed points are critical points governing a continuous phase transition. Since, at a continuous phase transition, the correlation length $\xi$ is infinite and the model is scale invariant.

The most crucial observation of Wilson is that in the RG transformation, i.e., the "coarse graining" process, new types of local interactions are generated and that the new interactions can be classified as either irrelevant or relevant interactions. Only relevant (including marginal) interactions are important in determining what kind of infrared dynamics characterized by a scale $\mu \sim 1 / \xi$ emerges from the microscopic or bare model characterized by the cutoff $\Lambda$. The effect of irrelevant interactions can always be absorbed by adapting the coupling constants of relevant and marginal interactions.

Especially, close to a continuous phase transition, the RG methods show that it is impossible, a priori (without solving the equations of motion), to determine which interactions are relevant or irrelevant; particular interactions can acquire anomalous dimensions and interactions which are irrelevant in a certain region of coupling constant space might become relevant in another region.

In this respect the GNJL model should be considered as the Wilsonian effective (or microscopic) action of QED taking into account the four-fermion interactions describing neutral scalar and pseudoscalar fermion-antifermion composites. It was shown in Refs. [17,18 that there is a critical line (curve) in the coupling constant plane $\left(\alpha_{0}, g_{0}\right)$ of the GNJL model separating a chiral symmetric phase from the chiral broken phase. The critical line is given by

$$
g_{c}\left(\alpha_{0}\right)=(1+\omega)^{2} / 4, \quad \omega=\sqrt{1-4 \lambda_{0}}, \quad \lambda_{0}=3 \alpha_{0} / 4 \pi .
$$

In the neighborhood of the critical line four-fermion interactions acquire sufficiently large anomalous dimensions to become relevant operators. The existence of nontrivial scaling behavior of the model near criticality implies that these scalar and pseudoscalar composites are relevant degrees of freedom at both short and long distances.[?

Lattice simulations of noncompact quenched QED with an induced four-fermion coupling were performed by the Illinois group in Refs. [19,20]. The Illinois group obtained a critical point $\left(0.44 \alpha_{c}, 0.76\right)$ in the $\left(\alpha_{0}, g_{0}\right)$ plane, which fits nicely on the critical line Eq. (田). However

${ }^{1}$ UV stable fixed points are specific roots of the $\beta$ functions.

${ }^{2}$ The composites are light states, as well in the symmetric phase as in the broken phase. 
it was not possible at that time to investigate by means of lattice simulations the phase transition along the critical line.

Later, the GNJL model was studied on the lattice in noncompact formulation using some mean-field approach for the fermions in Ref. [21]. They obtained a critical line qualitatively similar to the one following from the Schwinger-Dyson equation (SDE) approach Eq. (41).

In the intermediate region $\left(0<\alpha_{0}<\alpha_{c}\right)$, the critical exponents describing the chiral phase transition satisfy nonmean-field hyperscaling relations which supports the view that, within the quenched-ladder mean-field approximation, the GNJL model has a nontrivial continuum limit [19,22]. Also in Ref. [21] nonmean field critical exponents were obtained.

The physical implications and the consistency of the quenched-ladder results with many quenched lattice simulations, and with the nonperturbative RG techniques, support the view that the qualitative features of the approach might be realistic and describe properties of the full theory. Most likely this is due to the ladder approximation respecting the vector and axial Ward-Takahashi identities.

In Refs. 23, 24] the validity of the ladder approximation was tested positively by including the effects of, e.g., crossed photon exchange graphs. In addition, the nonperturbative renormalization-group methods of Refs. 225.26 provide a way to check the quenched-ladder approximation in the GNJL model by including the effect of crossed photon exchange graphs, and four-fermion interactions in the RG flow of coupling constants. In Ref. 26] the critical lines and critical exponents in the full quenched GNJL model were obtained in a particular so-called local potential approximation, which incorporates besides crossed photon exchange graphs also four-fermion exchanges beyond the mean-field approach. Considering the small quantitative differences, qualitatively this study supports the reliability of the ladder approximation.

Attempts to include a logarithmic running of the coupling drastically changes the chiral phase transition and the critical line, see Refs. 27 30. Moreover, it was shown in Refs. [30] 33] that the critical exponents are of the mean-field type (up to logarithmic violations) leading to a trivial theory.

Lattice simulations of noncompact full (unquenched) QED on the lattice (with flavors, $N=2$ and $N=4$ ) are controversial [34]. The Illinois group [35,36] (see also [37, 13]) and the Zaragosa group [38,39], find power-law scaling and nonmean-field critical exponents, signaling a possible nontrivial continuum limit for the strong-coupling broken phase, whereas 40,41,34 obtain mean-field behavior (mean-field critical exponents with logarithmic corrections). Thus Göckeler et al. find a vanishing renormalized gauge coupling and a vanishing effective Yukawa coupling (defined by the Goldberger-Treiman relation), and they conclude that lattice QED is trivial, see for their most recent result Ref. [42].

In this paper we argue that the four-fermion interactions might play a crucial role in the phenomenon of charge screening. We show that, by considering the Abelian GNJL model, UV stable fixed points of the $\beta$ function of the gauge coupling can be realized, provided the number of fermion flavors $N$ exceeds some critical value. The existence of UV stable fixed points gives rise to a nontrivial theory.

\footnotetext{
${ }^{3}$ They obtained the critical line: $g_{c}\left(\alpha_{0}\right)=(1+\omega)^{2} / 3$, which differs by a factor $3 / 4$ from the quenched-ladder result.
} 
An important observation is that the nonmean-field values for the critical exponents suggest the existence of a nontrivial Yukawa interaction describing the interactions of the scalar and pseudoscalar composites with fermions. This also points out the inconsistency of the mean-field approximation (Hartree-Fock approximation) for the four-fermion interactions. Therefore, we go beyond the mean-field approximation by incorporating these composites (the $\sigma$ and $\pi$ exchanges) in the $1 / N$ expansion.

The setup of this paper is the following. In Sec. II we introduce the Abelian GNJL model with $U_{L}(N) \times U_{R}(N)$ symmetry. Furthermore, we sketch how we will search for the existence of an UV stable fixed point in the coupling constant plane $\left(\alpha_{0}, g_{0}\right)$. In Sec. III we discuss, within the quenched-ladder mean-field approximation, the importance of hyperscaling relations and how this is related to the existence of a nontrivial Yukawa interaction in the GNJL model. We discuss how to proceed beyond the quenched mean-field approximation in Sec. IV. The $1 / N$ expansion is discussed in Sec. IVB. In order to get some idea how the scalar and pseudoscalar composites contribute to vacuum polarization we illustrate such contributions on the level of perturbation theory for a gauge-Higgs-Yukawa model in Sec. $\nabla$. Then, in Sec. VI, we argue how we can exploit the $1 / N$ expansion and derive a computable expression for the $\beta$ function, $\beta_{\alpha}$, of the gauge coupling. Subsequently, $\beta_{\alpha}$ is computed explicitly in Sec. VII. The existence of UV stable fixed points is addressed in Sec. VIII. Finally, we present our conclusions in Sec. IX].

\section{THE ABELIAN GNJL MODEL}

We consider the GNJL model with $U(1)$ gauge symmetry (the Abelian GNJL model) with $N$ number of fermion flavors described by the following Lagrangian (see also Refs. 43.44]):

$$
\mathcal{L}_{1}=\bar{\psi}_{i}\left(i \gamma^{\mu} D_{\mu}-m_{0}\right) \psi_{i}-\frac{1}{4} F_{\mu \nu} F^{\mu \nu}+\frac{G_{0}}{2} \sum_{\alpha=0}^{N^{2}-1}\left[\left(\bar{\psi}_{i} \tau_{i j}^{\alpha} \psi_{j}\right)^{2}+\left(\bar{\psi}_{i} \tau_{i j}^{\alpha}\left(i \gamma_{5}\right) \psi_{j}\right)^{2}\right]
$$

where $D_{\mu}=\partial_{\mu}-i e_{0} A_{\mu}$ and where the flavor labels $i, j$ run from 1 to $N$. The Lagrangian $\mathcal{L}_{1}$ is parametrized by the following three dimensionless bare coupling constants:

$$
\mu_{0}=m_{0} / \Lambda \equiv m_{\Lambda}, \quad \alpha_{0}=e_{0}^{2} / 4 \pi \equiv \alpha_{\Lambda}, \quad g_{0}=G_{0} \Lambda^{2} / 4 \pi^{2} \equiv g_{\Lambda},
$$

where $\Lambda$ is the ultraviolet cutoff. We assume that the above set of dimensionless coupling constants comprises the entire set of relevant (including marginal) dimensionless coupling constants respecting particular chiral, vector, and gauge symmetries.

If the bare mass $m_{0}$ is zero, $\mathcal{L}_{1}$ has a global $U_{L}(N) \times U_{R}(N)$ symmetry. The generators, $\tau$, of the $U(N)$ Lie algebra have the following properties:

$$
\tau^{\alpha \dagger}=\tau^{\alpha}, \quad \operatorname{Tr} \tau^{\alpha} \tau^{\beta}=\delta^{\alpha \beta}, \quad \sum_{\alpha=0}^{N^{2}-1} \tau_{i j}^{\alpha} \tau_{k l}^{\alpha}=\delta_{i l} \delta_{k j}
$$

The last identity is called the Fierz identity.

The Abelian GNJL model described by Eq. (5) can be conveniently analyzed in terms of auxiliary or composite fields $\sigma^{\alpha}=-G_{0} \bar{\psi} \tau^{\alpha} \psi$ and $\pi^{\alpha}=-G_{0} \bar{\psi} \tau^{\alpha}\left(i \gamma_{5}\right) \psi$ describing scalar and pseudoscalar degrees of freedom. In this way $\mathcal{L}_{1}$ can be rewritten as 


$$
\mathcal{L}_{2}=\bar{\psi}_{i} i \gamma^{\mu} D_{\mu} \psi_{i}-\frac{1}{4} F_{\mu \nu} F^{\mu \nu}-\sum_{\alpha=0}^{N^{2}-1} \bar{\psi}_{i} \tau_{i j}^{\alpha}\left(\sigma^{\alpha}+i \gamma_{5} \pi^{\alpha}\right) \psi_{j}-\frac{1}{2 G_{0}} \sum_{\alpha=0}^{N^{2}-1}\left[\left(\sigma^{\alpha}\right)^{2}+\left(\pi^{\alpha}\right)^{2}\right]
$$

where $m_{0}$ has been set to zero. In this formulation the four-fermion interactions are described by the interactions of the auxiliary fields with the fermion fields. Then the connected twopoint Green functions of the $\pi^{\alpha}$ fields describe $N^{2} \mathrm{Nambu}-$ Goldstone bosons ( $\pi$ bosons) and the connected two-point Green functions of the $\sigma^{\alpha}$ fields describe $N^{2}$ "Higgs" bosons $(\sigma$ bosons).

Although the Lagrangian (5) has very interesting properties, surprisingly this particular class of GNJL models with $U_{L}(N) \times U_{R}(N)$ has not received much attention. One such property is that Eq. (5) comprises the largest set of relevant chiral invariant four-fermion operators for a model of $N$ fermions. Another independent set of vectorlike chiral invariant four-fermion interactions such as $\left(\bar{\psi} \gamma^{\mu} \psi\right)^{2}$ do not acquire large anomalous dimensions and remain irrelevant near the critical point (line). This has been shown in Refs. 25, 26], where the RG flow of scalarlike chiral invariant and vectorlike chiral invariant four-fermion interactions has been considered. Moreover, the specific form of the chiral symmetry, where the number of scalars equals the number of pseudoscalars, turns out to have useful implications in the context of $1 / N$ expansions as we will discuss later in Sec. IV.

In case of $\mathcal{L}_{2}$ there are four renormalization constants:

$$
\begin{aligned}
& Z_{2}^{1 / 2}\left(\Lambda^{\prime} / \Lambda\right) \psi_{\left(\Lambda^{\prime}\right)}(x)=\psi(x), \quad Z_{3}^{1 / 2}\left(\Lambda^{\prime} / \Lambda\right) A_{\left(\Lambda^{\prime}\right)}^{\mu}(x)=A^{\mu}(x), \\
& Z_{\sigma}\left(\Lambda^{\prime} / \Lambda\right) \sigma_{\left(\Lambda^{\prime}\right)}(x)=\sigma(x), \quad Z_{\pi}\left(\Lambda^{\prime} / \Lambda\right) \pi_{\left(\Lambda^{\prime}\right)}(x)=\pi(x),
\end{aligned}
$$

where $\Lambda^{\prime} / \Lambda \leq 1$, and the fields $\psi, A^{\mu}, \pi, \sigma$ are the bare fields defined at the UV cutoff $\Lambda$.

Near the critical point a new scale is generated: the correlation length $\xi$. In case of a second-order type of phase transition, the correlation length exists in both phases of the system. In the broken phase the inverse correlation length is real and can be considered as a physical mass of particles, for instance the mass of the scalar bound state (the $\sigma$ boson) $m_{\sigma}$, or the mass of fermion $m_{\mathrm{dyn}}$. In the symmetric phase the fermion is massless, and the scalar and pseudoscalar composites are unstable states characterized by a complex mass pole in their respective propagators describing the mass and the width of the Breit-Wigner type resonance, see Refs. [45 47]. The absolute value of the complex mass pole $\left|m_{\sigma}\right|$ can be considered as the inverse correlation length, i.e., $\left|m_{\sigma}\right| \sim 1 / \xi$.

The RG transformation dictates the flow of the dimensionless bare couplings as function of the UV cutoff $\Lambda$. Typically the bare relevant and marginal couplings $\left(e . g ., \mu_{0}, \alpha_{0}, g_{0}\right)$ have to be fine-tuned sufficiently close to the critical point in order for scaling behavior to set in, so that the physics in the infrared can be related to experimental data. Scaling behavior is obtained when there is a large scale hierarchy between the infrared length scale $\xi$ and the ultraviolet length scale $a=1 / \Lambda$, i.e., $\xi \gg a$.

The fine-tuning depends on the eigenvalues of the RG transformation of the couplings close to the critical point and hence on the critical exponents. These critical exponents can be derived from the $\beta$ functions for the coupling constants $\mu_{0}, \alpha_{0}$, and $g_{0}$;

$$
\Lambda \frac{d \mu_{0}}{d \Lambda}=\beta_{\mu}\left(\mu_{0}, \alpha_{0}, g_{0}\right), \quad \Lambda \frac{d \alpha_{0}}{d \Lambda}=\beta_{\alpha}\left(\mu_{0}, \alpha_{0}, g_{0}\right), \quad \Lambda \frac{d g_{0}}{d \Lambda}=\beta_{g}\left(\mu_{0}, \alpha_{0}, g_{0}\right) .
$$

The crucial step is determine the fixed points $\left(\mu_{\star}, \alpha_{\star}, g_{\star}\right)$ of the RG equations (11), i.e., 


$$
\beta_{\mu}\left(\mu_{\star}, \alpha_{\star}, g_{\star}\right)=0, \quad \beta_{\alpha}\left(\mu_{\star}, \alpha_{\star}, g_{\star}\right)=0, \quad \beta_{g}\left(\mu_{\star}, \alpha_{\star}, g_{\star}\right)=0,
$$

since the nature of the fixed point determines whether a nontrivial continuum limit can be realized or not. For a nontrivial continuum limit $\left(\mu_{\star}, \alpha_{\star}, g_{\star}\right)$ should be a UV stable fixed point.

The RG equations follow from the regularized SDE's of the generating functional described by the Lagrangian (8). I The UV stable fixed point for $\beta_{\mu}$ is $\mu_{\star}=0$, hence we can write

$$
\Lambda \frac{d \mu_{0}}{d \Lambda} \approx-\left(1+\gamma_{m}\right) \mu_{0}
$$

where $\gamma_{m}$ is the anomalous dimension of the mass operator $\bar{\psi} \psi$ evaluated at the fixed point $\left(\mu_{\star}, \alpha_{\star}, g_{\star}\right)$. In the case of the quenched GNJL models $1 \leq \gamma_{m} \leq 2$. Thus the dimensionless bare mass $\mu_{0}$ is a relevant coupling requiring fine-tuning.

After setting $\mu_{0}=\mu_{\star}=0$, the problem reduces to the determination of the UV stable fixed points in the coupling constant plane $\left(\alpha_{0}, g_{0}\right)$ : i.e.,

$$
\begin{array}{ll}
\beta_{g}\left(\alpha_{\star}, g_{\star}\right)=0, & \beta_{g}^{\prime}\left(\alpha_{\star}, g_{\star}\right)=\left.\frac{\partial \beta_{g}(\alpha, g)}{\partial g}\right|_{(\alpha, g)=\left(\alpha_{\star}, g_{\star}\right)}<0, \\
\beta_{\alpha}\left(\alpha_{\star}, g_{\star}\right)=0, & \beta_{\alpha}^{\prime}\left(\alpha_{\star}, g_{\star}\right)=\left.\frac{\partial \beta_{\alpha}(\alpha, g)}{\partial \alpha}\right|_{(\alpha, g)=\left(\alpha_{\star}, g_{\star}\right)}<0 .
\end{array}
$$

The quenched-ladder approximation simplifies the solutions of Eqs. (14) and (15) considerably since the quenched hypothesis explicitly sets $\beta_{\alpha}=0$ for all $\alpha_{0}$ by omiting fermion loops. It was shown in Ref. [48] that, in the symmetric phase $\left(g_{0} \leq g_{c}\right)$,

$$
\beta_{g}\left(\alpha_{0}, g_{0}\right)=-2 \omega \frac{g_{0}}{g_{c}}\left(g_{0}-g_{c}\right),
$$

with $\omega$ and $g_{c}$ given in Eq. (4). In the next section, Eq. (16) will be derived. Clearly, in this way, the UV stable fixed point of $\beta_{g}$ is the critical line;

$$
g_{\star}=g_{c}\left(\alpha_{0}\right), \quad \beta_{g}\left(\alpha_{0}, g_{c}\left(\alpha_{0}\right)\right)=0 .
$$

Now Eq. (15) should be reconsidered. We will analyze Eq. (15) beyond the quenched approximation, and try to solve

$$
\beta_{\alpha}\left(\alpha_{0}, g_{c}\left(\alpha_{0}\right)\right)=0
$$

In Sec. VI an explicit expression for $\beta_{\alpha}$ will be derived by assuming that $g_{0}$ is at its critical value $g_{c}$ and that it has an UV stable fixed point so that in the neighborhood of this point $\beta_{\alpha} \approx 0$.

\footnotetext{
${ }^{4}$ A derivation of the set of (full) SDE's for the two and three-point functions is given in Chap. 2 of 44.
} 


\section{HYPERSCALING IN THE QUENCHED-LADDER MEAN-FIELD APPROXIMATION}

In analogy with statistical mechanics, the continuous chiral phase transition can be classified in terms of critical exponents which describe the scaling of various macroscopic quantities (e.g., the chiral condensate, correlation length, effective potential, chiral susceptibility) close to or at the critical point. It is considered a strong indication of the existence of a nontrivial continuum limit $(\Lambda \rightarrow \infty)$, if so-called hyperscaling relations between these various critical exponents are satisfied, see Refs. [19,22,49 51].

Because the $\sigma$ boson propagator $\Delta_{S}$ is the connected correlation function of the field $\sigma$ describing correlations parallel to direction of symmetry breaking (i.e., parallel to the direction of long-range ordering), the absolute value of the mass, $m_{\sigma}$, of the $\sigma$ boson, which is given by $\Delta_{S}$, is the natural candidate for the inverse correlation length.

In the quenched-ladder approximation treating four-fermion interaction in a mean-field approximation, the critical exponents are

$$
\delta=\frac{2+\omega}{2-\omega}, \quad \beta=\frac{2-\omega}{2 \omega}, \quad \nu=\frac{1}{2 \omega}, \quad \gamma=1
$$

and satisfy the hyperscaling relations

$$
\gamma=\beta(\delta-1), \quad 4 \nu=2 \beta+\gamma
$$

Other hyperscaling relations involving the critical exponent $\alpha$ describing the scaling of the effective potential are satisfied too [19,22]. Furthermore, it was argued in [19] that the validity of the quenched-ladder mean-field approximation relies on the verification that the critical exponent $\gamma=1$. The interpretation of $\gamma=1$ is the factorization $\eta_{(\bar{\psi} \psi)^{2}}=2 \eta_{(\bar{\psi} \psi)}$. A renormalization of the chiral condensate simultaneously renormalizes the propagators $\Delta_{S}$ and $\Delta_{P}$. Indeed the lattice computations of the critical exponent $\gamma$ reported in [19 21] showed strong evidence for $\gamma=1$.

The anomalous dimension $\eta$ describes the scaling of the connected two-point Green function $\Delta_{S}$ at the critical point. In Ref. 47 the scalar propagator $\Delta_{S}$ (see Fig. 2) and the scalar Yukawa vertex $\Gamma_{S}$ (see Fig. 3) have been computed in the symmetric phase in the quenched-ladder approximation by means of a so-called two-channel approximation.

In the symmetric phase the Yukawa vertex has the following form:

$$
\Gamma_{S}(p+q, p)=\mathbf{1}\left[F_{1}(p+q, p)+(\hat{q} \hat{p}-\hat{p} \hat{q}) F_{2}(p+q, p)\right] .
$$

These vertex functions $F_{1}$ and $F_{2}$ can be expanded in terms of Chebyshev polynomials of the second kind, e.g.,

$$
F_{1}(p+q, p)=\sum_{n=0}^{\infty} f_{n}\left(p^{2}, q^{2}\right) U_{n}(\cos \alpha), \quad \cos \alpha=\frac{p \cdot q}{p q} .
$$

The two-channel approximation of Ref. [47] now holds in that the Yukawa vertex is approximated by the angular average of the vertex function $F_{1}$ in the following way:

$$
\Gamma_{S}(p+q, p) \approx \mathbf{1} \int \frac{d \Omega_{p}}{2 \pi^{2}} F_{1}(p+q, p)=\mathbf{1} f_{0}\left(p^{2}, q^{2}\right)
$$


where

$$
f_{0}\left(p^{2}, q^{2}\right) \equiv F_{\mathrm{IR}}\left(p^{2}, q^{2}\right) \theta\left(q^{2}-p^{2}\right)+F_{\mathrm{UV}}\left(p^{2}, q^{2}\right) \theta\left(p^{2}-q^{2}\right) .
$$

The functions $F_{\mathrm{IR}}$ and $F_{\mathrm{UV}}$ are, respectively, referred to as the IR channel (infrared), and the UV channel (ultraviolet). The specific choice of Chebyshev expansion and consequently, the choice of zeroth-order coefficient $f_{0}$, is convenient, since the infrared limit $\left(q^{2} \gg p^{2}\right)$, and ultraviolet limit $\left(q^{2} \ll p^{2}\right)$ of $\Gamma_{S}$ are both described by $f_{0}$. The scaling form for $\Delta_{S}(q)$ is well described by these two functions $F_{\mathrm{IR}}$ and $F_{\mathrm{UV}}$.

The zeroth-order Chebyshev expansion or two-channel approximation gives second-order differential equations for the lowest order harmonic with appropriate IR and UV boundary conditions. These differential equations are exactly solvable and the solutions are expressed in terms of a Bessel function of the first kind for $F_{\mathrm{IR}}$ and in terms of modified Bessel functions for $F_{\mathrm{UV}}$. The solutions are given in [47].

With the "asymptotic solutions" for $\Gamma_{S}$ given in terms of $F_{\mathrm{IR}}$ and $F_{\mathrm{UV}}$, an analytic expression for the $\sigma$ boson can be obtained from

$$
\Pi_{S}\left(q^{2}\right)=\frac{\Lambda^{2}}{4 \pi^{2}} \frac{1}{\lambda_{0}}\left[F_{\mathrm{UV}}\left(\Lambda^{2}, q^{2}\right)-1\right], \quad \Delta_{S}^{-1}(q)=-\frac{1}{G_{0}}+\Pi_{S}\left(q^{2}\right),
$$

which has been argued in 47 to be correct up to leading and next-to-leading order in $q^{2} / \Lambda^{2}$ and is valid along the entire critical curve $g_{c}$.

Also one can derive that the solutions for $\Delta_{S}$ and are consistent with hyperscaling for $0<\alpha_{0}<\alpha_{c}$. This is intimately related to the fact that the renormalization of the auxiliary fields $\sigma$ and $\pi$, Eq. (10), simultaneously renormalizes the Yukawa vertex and the scalar propagator.

Near $g_{0}=g_{c}$ (with $\left|m_{\sigma}\right|^{2}, q^{2} \ll \Lambda^{2}$ ) the scalar propagator $\Delta_{S}$ has the scaling form 44,49 51 (in Euclidean formulation):

$$
\Delta_{S}(q)=\frac{1}{\Lambda^{2}}\left(\frac{\Lambda^{2}}{q^{2}}\right)^{1-\eta / 2} \mathcal{F}_{\Delta}\left(\left|m_{\sigma}\right|^{2} / q^{2}\right), \quad \mathcal{F}_{\Delta}(x) \approx-\frac{4 \pi^{2}}{B(\omega)} \frac{1}{1+x^{\omega}},
$$

where $\eta$ is the anomalous dimension

$$
\eta=2(1-\omega)
$$

and where

$$
\begin{aligned}
B(\omega) & \equiv \frac{16 \omega}{\left(1-\omega^{2}\right)^{2}} \frac{\gamma(-\omega)}{\gamma(\omega)} \frac{\Gamma(2-\omega)}{\Gamma(2+\omega)}\left(\frac{\lambda_{0}}{2}\right)^{\omega}, \\
\gamma(\omega) & \equiv \sqrt{2 \lambda_{0}}\left[J_{1}\left(\sqrt{2 \lambda_{0}}\right) I_{\omega}^{\prime}\left(\sqrt{2 \lambda_{0}}\right)+J_{1}^{\prime}\left(\sqrt{2 \lambda_{0}}\right) I_{\omega}\left(\sqrt{2 \lambda_{0}}\right)\right] .
\end{aligned}
$$

With Eq. (19), the anomalous dimension $\eta$, Eq. (27), satisfies the hyperscaling relation

$$
\gamma=\nu(2-\eta) \text {. }
$$

It has been shown in [45,47] that $\Delta_{S}$ has a complex pole on a second Riemann sheet in (Minkowsky) momentum space. The mass $m_{\sigma}$ is the complex pole of $\Delta_{S}$ and the absolute value $\left|m_{\sigma}\right|$ scales according to $\left|m_{\sigma}\right| \sim\left(\Delta g_{0}\right)^{\nu}$. More precisely, in 45,47] it is derived that 


$$
\left|m_{\sigma}\right| \propto \Lambda\left[\frac{-\Delta g_{0}}{g_{c} g_{0} B(\omega)}\right]^{1 / 2 \omega}, \quad \Delta g_{0}=g_{0}-g_{c} .
$$

The absolute value of $m_{\sigma}$ is taken to be the physical, macroscopic or infrared mass scale, which by definition should be independent of $\Lambda$. Using Eq. (31), we can derive the $\beta$ function of $g_{0}$ by assuming that

$$
0=\Lambda \frac{d\left|m_{\sigma}\right|}{d \Lambda} \Longrightarrow \beta_{g}\left(\alpha_{0}, g_{0}\right)=-2 \omega \frac{g_{0}}{g_{c}}\left(g_{0}-g_{c}\right)
$$

which is equivalent to the $\beta$ function given in Ref. 48 and Sec. II. Hence the critical curve $g_{0}=g_{c}$ is an UV stable fixed point $\beta_{g}\left(\alpha_{0}, g_{c}\right)=0\left(\beta_{g}^{\prime}\left(\alpha_{0}, g_{c}\right)<0\right)$ of the RG flow.

In accordance with Ref. [47], the scaling form for the Yukawa vertex can be written as

$$
\Gamma_{S}(p+q, p) \approx \mathbf{1}\left(\frac{\Lambda^{2}}{q^{2}}\right)^{\eta / 4}\left[\mathcal{F}_{\mathrm{IR}}\left(p^{2} / q^{2}\right) \theta\left(q^{2}-p^{2}\right)+\mathcal{F}_{\mathrm{UV}}\left(q^{2} / p^{2}\right) \theta\left(p^{2}-q^{2}\right)\right]
$$

where, for $p^{2}, q^{2} \ll \Lambda^{2}$,

$$
F_{\mathrm{IR}}\left(p^{2}, q^{2}\right) \approx\left(\frac{\Lambda^{2}}{q^{2}}\right)^{\eta / 4} \mathcal{F}_{\mathrm{IR}}\left(p^{2} / q^{2}\right), \quad F_{\mathrm{UV}}\left(p^{2}, q^{2}\right) \approx\left(\frac{\Lambda^{2}}{q^{2}}\right)^{\eta / 4} \mathcal{F}_{\mathrm{UV}}\left(q^{2} / p^{2}\right)
$$

and

$$
\begin{aligned}
\mathcal{F}_{\mathrm{IR}}\left(p^{2} / q^{2}\right) & =\frac{2 \sin \omega \pi}{\pi} \frac{2}{\gamma(\omega)} \frac{\Gamma(1-\omega)}{(1+\omega)}\left(\frac{\lambda_{0}}{2}\right)^{\omega / 2}\left(\frac{q^{2}}{p^{2}}\right)^{1 / 2} J_{1}\left(\sqrt{\frac{2 \lambda_{0} p^{2}}{q^{2}}}\right) \\
\mathcal{F}_{\mathrm{UV}}\left(q^{2} / p^{2}\right) & =\frac{2}{\gamma(\omega)} \frac{\Gamma(1-\omega)}{(1+\omega)}\left(\frac{\lambda_{0}}{2}\right)^{\omega / 2}\left(\frac{q^{2}}{p^{2}}\right)^{1 / 2} \\
& \times\left[\gamma(\omega) I_{-\omega}\left(\sqrt{\frac{2 \lambda_{0} q^{2}}{p^{2}}}\right)-\gamma(-\omega) I_{\omega}\left(\sqrt{\frac{2 \lambda_{0} q^{2}}{p^{2}}}\right)\right] .
\end{aligned}
$$

From the above scaling form for $\Delta_{S}$ and $\Gamma_{S}$ it is clear that four-fermion scattering amplitudes such as

$$
\Gamma_{S}\left(p_{1}+q, p_{1}\right) \Delta_{S}(q) \Gamma_{S}\left(p_{2}, p_{2}+q\right) \propto \frac{1}{q^{2}}, \quad p_{1}^{2}, p_{2}^{2} \ll q^{2} \ll \Lambda^{2},
$$

are independent of $\Lambda$ and express the long-range naturef of Yukawa forces. For dimensions $2<d<4$, this was pointed out in Ref. 46]. The long-range Yukawa forces and their nontrivial contributions to scattering amplitudes in the infrared are a direct consequence of hyperscaling and thus powerlike renormalizability.

The consensus is that in four dimensions due to the logarithmic corrections, the hyperscaling relations are violated for the pure NJL model and $\lambda \phi^{4}$ theory, and we have the following inequalities:

\footnotetext{
${ }^{5}$ The correlation length is large, $1 /\left|m_{\sigma}\right| \gg 1 / \Lambda$.
} 


$$
4 \nu>2 \beta+\gamma, \quad \gamma>\beta(\delta-1)
$$

see Ref. 52 for an extensive discussion regarding this issue. This violation of hyperscaling is believed to be a sign of triviality meaning that the effective Yukawa coupling (which couples Goldstone bosons to the fermions) vanishes in the continuum limit. The continuum limit is noninteracting, hence trivial. This can be seen in the following way. Assuming that in the low-energy region the correlation length $\xi$ is the only relevant length scale, we define an effective Yukawa coupling $g_{Y}$ by the zero-momentum limit of the four-fermion scattering amplitude (two fermions exchanging a scalar bound state) in the D $\chi \mathrm{SB}$ phase

$$
\frac{g_{Y}^{2}}{m_{\sigma}^{2}} \sim \xi^{2} g_{Y}^{2} \sim \Gamma_{S}(0,0) \Delta_{S}(0) \Gamma_{S}(0,0)
$$

where $\Gamma_{S}(0,0)$ and $\Delta_{S}(0)$ are given by the chiral susceptibility relations

$$
\Delta_{S}(0)=-G_{0} \frac{\partial\langle\sigma\rangle}{\partial m_{0}}, \quad \Gamma_{S}(p, p)=(\mathbf{1}) \frac{\partial \Sigma\left(p^{2}\right)}{\partial\langle\sigma\rangle}
$$

where $\Sigma_{0}=\Sigma(0)$ is the fermion mass in the broken phase. By making use of the scaling laws [19,22] and that $\xi \sim 1 / \Sigma_{0} \sim 1 / m_{\sigma},\langle\sigma\rangle \sim\langle\bar{\psi} \psi\rangle$, we can derive that

$$
g_{Y}^{2} \sim \xi^{(2 \beta+\gamma-4 \nu) / \nu}
$$

This expression is related to the definition of $g_{R} \sim g_{Y}^{2}$ given in Ref. [52, and it is clear that the scaling inequalities (38) imply that $g_{Y}^{2} \rightarrow 0$ when $\xi \rightarrow \infty$. Thus, only if the hyperscaling relations $(20)$ are satisfied, a nonzero $g_{Y}$ might be realized in the continuum limit $(\xi \rightarrow \infty)$, thereby giving rise to a nontrivial interacting theory.

\section{BEYOND THE QUENCHED-LADDER MEAN-FIELD APPROXIMATION}

The quenched approximation is analogous to the assumption that the full photon propagator

$$
D_{\mu \nu}(q)=\left(-g_{\mu \nu}+\frac{q_{\mu} q_{\nu}}{q^{2}}\right) \Delta(q)-a \frac{q_{\mu} q_{\nu}}{q^{4}}, \quad \Delta(q) \equiv \frac{1}{q^{2}} \frac{1}{1+\Pi\left(q^{2}\right)}
$$

can be approximated by the bare or canonical propagator $\Delta(q)=1 / q^{2}$ (for large momenta),

$$
D_{\mu \nu}(q)=\left(-g_{\mu \nu}+\frac{q_{\mu} q_{\nu}}{q^{2}}\right) \frac{1}{q^{2}},
$$

in the Landau gauge $(a=0)$. The quenched approximation is only consistent when the vacuum polarization is finite in the continuum limit, i.e., the logarithmic running of the coupling is absent. This is the case at an UV stable fixed point of the $\beta$ function, Eq. (15), of $\alpha_{0}$. The assumption that such a critical fixed point exists, and that it lies somewhere on the critical curve (田) is the starting point for many studies of dynamical chiral symmetry breaking in context of the GNJL model. In fact, the quenched hypothesis (43) is only 
consistent when the bare gauge coupling $\alpha_{0}$ is near the fixed point of the theory, $\beta_{\alpha} \approx 0$. We discuss this issue in more detail in Sec. V1.

In many approximations of the GNJL model, the four-fermion interactions are treated in a mean-field approach known as the Hartree-Fock approximation. In mean-field approximations the composite operators such as $\bar{\psi} \psi$ are replaced by their vacuum expectation values $(\bar{\psi} \psi \rightarrow\langle\bar{\psi} \psi\rangle)$ and fluctuations about that value are ignored. Thus quantum corrections corresponding to four-fermion interactions are neglected beyond tree level.

As long as four-fermion interactions are irrelevant the mean-field approach for these operators is justified. However, in Refs. [19,22] it is concluded that, within the quenchedladder mean-field approximation to the GNJL model, the hyperscaling equations for the critical exponents are satisfied, implying that the four-fermion operators become relevant due to the appearance of large anomalous dimensions. In other words, the mean-field approach yields non-mean-field exponents, thereby being inconsistent (e.g., see Refs. [21,50]). As was discussed in Sec. III, the hyperscaling relations imply the existence of a nontrivial Yukawa interaction describing the interaction between fermions and $\sigma$ and $\pi$ composites in the GNJL model at both short and long distances.

In order to go beyond the mean-field approach, we propose the following. First, we point out the usefulness of skeleton expansions. Second, we make use of the specific form of the chiral symmetry and adopt the $1 / N$ expansion (with $N$ the number of fermion flavors).

\section{A. The skeleton expansion}

The non-mean-field values of the critical exponents imply that one cannot neglect (as is done in mean-field approximations) the full connected Green functions corresponding to the composites (or at least the leading or asymptotic parts of these functions) in the SDE's.

On the level of the Bethe-Salpeter (BS) fermion-antifermion scattering kernels the $\sigma$ and $\pi$ composites can be incorporated in a RG invariant manner by making use of the skeleton expansion, e.g., see Ref. [53]). Analogous to QED kernels we define the one-boson irreducible kernel $K^{(1)}$, and the two-fermion one-boson irreducible BS kernel $K^{(2)}$, where these kernels now also include the $\sigma$ and $\pi$ composites. For both type of kernels a skeleton expansion exists. The integral equation between $K^{(1)}$ and $K^{(2)}$ is known as the Bethe-Salpeter equation.

The skeleton expansion is a series in topologically distinct Feynman diagrams with all vertices and propagators fully dressed. The skeleton expansion is a special way of resumming the entire set of Feynman diagrams in a consistent manner, i.e., without double counting. The lowest order terms ("lowest" in terms of loops) of the skeleton expansion for $K^{(2)}$ is illustrated in Fig. 4. The blobs with the letter "B" in the full vertices and propagators represent photons, and composite $\sigma$ and $\pi$ exchanges.

Each term in the skeleton expansion of the BS kernel $K^{(2)}$ is RG invariant, up to fermion wave function factors, i.e. the expansion is independent of the renormalization factors $Z_{3}$ and $Z=Z_{\sigma}=Z_{\pi}$ (see Eqs. (9) and (10)) of, respectively, the gauge field and the composite fields $\sigma$ and $\pi$. The two $Z^{-1}$ factors with anomalous dimensions of each Yukawa vertex cancel with the $Z^{2}$ factors of the $\sigma$ and $\pi$ propagators, leading to cutoff independent fermion-antifermion scattering amplitudes, e.g., see Eq. (37). 


\section{B. The $U_{L}(N) \times U_{R}(N)$ chiral symmetry and the $1 / N$ expansion}

As was mentioned in Sec. [II, the Abelian GNJL model, with $N$ number of fermion flavors, is taken to be invariant under global $U_{L}(N) \times U_{R}(N)$ chiral transformations, so that both the scalar and pseudoscalar four-fermion interactions are in the adjoint representation, and, consequently, the number of scalar composites $\left(N^{2}\right)$ equals the number of pseudoscalar composites $\left(N^{2}\right)$. In this way, when $N$ is large we can use the $1 / N$ expansion introduced by 't Hooft [54. This provides us with a scheme to incorporate four-fermion interactions beyond the mean-field approach. The $1 / N$ expansion states that the planar (i.e., ladder) diagrams, with fermions at the edges, describe the leading or dominant contributions to Green functions.

The interesting feature of such a $1 / N$ expansion is that Feynman diagrams can be classified in terms of two-dimensional surfaces with specific topology. Diagrams with other (than planar) topological structures are suppressed by at least factors of $1 / N$, and in the limit of large $N$, their contribution can be neglected with respect to planar graphs. One important rule is to draw Feynman graphs with fermion loops forming the boundary of the graph (if possible). In this way, vertex corrections are not necessarily classified as being planar.

In the context of the 't Hooft's $1 / N$ expansion, we should consider internal or virtual $\sigma$ and $\pi$ exchanges analogous to the gluon exchanges with the important difference that due to the chiral symmetry we have two types of particles both being in the adjoint representation ( $N^{2}$ scalars and $N^{2}$ pseudoscalars). Then, by keeping track of the flavor indices within a particular Feynman diagram, we can count factors of $1 / N$. Each fermion carries a flavor index $(i)$, which runs from 1 to $N$. A virtual $\sigma, \pi$ exchange, being associated with two Yukawa vertices, carries two flavor indices. Therefore, as a result of the Fierz identity (7), each virtual $\sigma, \pi$ exchange gives rise to a pair of Kronecker $\delta$ functions connecting the flavor indices of the scattered fermions. In the context of flavor indices, either a $\sigma$ or $\pi$ boson can be considered as a propagating fermion-antifermion pair carrying double flavor indices.

Whenever a trace over a flavor Kronecker $\delta$ function enters into the expression for a particular Feynman diagram, we speak of an index loop. An index loop is easily identified by using the double-line representation of 't Hooft. A fermion propagator is represented by a single index line (i.e., fermion line), whereas each internal scalar, respectively, pseudoscalar propagator is represented by a double index line. Consequently, whenever, after drawing a particular Feynman diagram, an index line closes, it forms an index loop giving rise to a factor $N=\operatorname{Tr} \delta$.

The topology of the Feynman diagram determines the factors of $N$. The vacuum polarization has the topology of a sphere with a single hole (i.e., a disk), where the fermion-loop forms the boundary (i.e., hole) of the graph. It can be shown straightforwardly, that planar diagrams in the vacuum polarization with $n$ exchanges of $\sigma$ 's and $\pi$ 's are associated with a factor $N^{n+1} g_{Y}^{2 n}$, where $g_{Y}$ is an "effective" Yukawa coupling describing the interaction of scalars and pseudoscalar with fermions. For the time being we leave unspecified such a coupling.

In absence of bare mass, the $U_{L}(N) \times U_{R}(N)$ symmetry allows us to write each full Yukawa vertex, photon-fermion vertex, fermion propagator, and $\sigma, \pi$ boson propagator as

$$
\Gamma_{S_{i j}^{a b}}^{\alpha}(k, p)=\tau_{i j}^{\alpha} \Gamma_{S a b}(k, p), \quad \Delta_{S}^{(\alpha)}(q)=\Delta_{S}(q),
$$




$$
\begin{aligned}
& \Gamma_{P_{i j}^{a b}}^{\alpha}(k, p)=\tau_{i j}^{\alpha} \Gamma_{P a b}(k, p), \quad \Delta_{P}^{(\alpha)}(q)=\Delta_{P}(q), \\
& \Gamma_{\substack{a b \\
i j}}^{\mu}(k, p)=\delta_{i j} \Gamma_{a b}^{\mu}(k, p), \quad S^{(i)}(p)=S(p),
\end{aligned}
$$

with $a, b$ spinor indices, $i, j$ flavor indices, and $\alpha$ the $U(N)$-generator index (see Refs. 44,47] for the definitions of the proper vertices and connected two-point Green functions). So that

$$
\sum_{\alpha=0}^{N^{2}-1} \Gamma_{S_{i j}^{a b}}^{\alpha}(k+q, k) \Delta_{S}^{(\alpha)}(q) \Gamma_{S_{k l}^{c d}}^{\alpha}(p, p+q)=\delta_{i l} \delta_{k j} \Gamma_{S a b}(k+q, k) \Delta_{S}(q) \Gamma_{S c d}(p, p+q),
$$

because of the Fierz identity (7). Then the first term of the skeleton expansion for $K^{(2)}$ is the following single boson exchange term:

$$
\begin{aligned}
\left(-i e_{0}^{2}\right) K_{\substack{a b, c d \\
i_{1} j_{1}, i_{2} j_{2}}}^{(2)}(k, p, p+q) & =\delta_{i_{1} j_{1}} \delta_{i_{2} j_{2}}(-i) \Gamma_{S c b}(p+q, p) i \Delta_{S}(q)(-i) \Gamma_{S a d}(k, k+q) \\
& +\delta_{i_{1} j_{1}} \delta_{i_{2} j_{2}}(-i) \Gamma_{P c b}(p+q, p) i \Delta_{P}(q)(-i) \Gamma_{P a d}(k, k+q) \\
& +\delta_{i_{2} j_{1}} \delta_{i_{1} j_{2}}\left(-i e_{0}\right) \Gamma_{c b}^{\lambda}(p+q, p) i D_{\lambda \sigma}(q)\left(-i e_{0}\right) \Gamma_{a d}^{\sigma}(k, k+q) .
\end{aligned}
$$

As a result of the chiral symmetry the contributions of four-fermion interactions, which are represented by $\sigma$ and $\pi$ exchanges, exhibit two distinct features depending on whether they are incorporated in SDE's describing quantities connected with so-called zero-spin structures (e.g., the dynamical mass $\Sigma$, the Yukawa vertices $\Gamma_{S}, \Gamma_{P}$, and the $\sigma$ and $\pi$ propagators $\left.\Delta_{S}, \Delta_{P}\right)$, or whether the exchanges are included in SDE's describing nonzero-spin structures (anti-commuting with $\gamma_{5}$ ) (e.g., the vacuum polarization $\Pi$, the photon-fermion vertex $\Gamma^{\mu}$, and the fermion wave function $\mathcal{Z}=Z_{2}$ ). Henceforth, we refer to (non)zero-spin functions, and their equations as (non)zero-spin channels.

The chiral symmetry gives rise to the following properties.

1. In spin-zero-channels, the contribution of planar diagrams (i.e., planar in $\sigma$ and $\pi$ exchanges) vanishes due to the fact that the exchange of a $\sigma$ has an opposite sign with respect to a $\pi$ exchange. Why? Let us consider a planar contribution to the scalar vacuum polarization which contains (amongst others) a $\pi$ exchange. Both $\gamma_{5}$ matrices corresponding to this particular planar $\pi$ exchange can be eliminated from the fermion trace of the scalar vacuum polarization by moving them to the right-hand side of the trace. For planar diagrams such a process involves the interchange of the $\gamma_{5}$ matrix with an even number of fermion propagators, and an arbitrary number of Yukawa vertices. Since the Yukawa vertices commute with the $\gamma_{5}$ matrix, and $\gamma_{5}$ anticommutes with the fermion propagator $S$, the process of moving the $\gamma_{5}$ to the right does not introduce an overall minus sign. Now using that $\left(i \gamma_{5}\right)\left(i \gamma_{5}\right)=-(\mathbf{1})(\mathbf{1})$, we see that the diagram containing a specific planar $\pi$ exchange is identical to minus the same diagram with the $\pi$ exchange replaced by a $\sigma$ exchange. Since each diagram containing a $\pi$ exchange has a scalar counter part (i.e., an analogous diagram with a

\footnotetext{
${ }^{6}$ Such structures are characterized by spinor matrices which commute with the $\gamma_{5}$ matrix.

${ }^{7}$ In the symmetric phase $\gamma_{5} S=-S \gamma_{5}$.
} 
$\sigma$ instead of a $\pi$ exchange), the sum of all planar diagrams, with a particular number of exchanges, vanishes.

2. In nonzero-spin channels (think of $\Pi, \Gamma^{\mu}$, etc.) containing vertices which anti-commute with the $\gamma_{5}$ matrix, the situation is different: planar $\sigma$ and $\pi$ exchanges contribute with identical sign. Let us now consider a planar contribution to the (photon) vacuum polarization containing a $\pi$ exchange. If we again move the $\gamma_{5}$ matrices to the righthand side of the trace, we get an overall minus sign due to the anti-commutation of $\gamma_{5}$ with $\gamma^{\mu}$. This means that any planar diagram in the vacuum polarization containing a $\pi$ exchange is identical to the same diagram with the $\pi$ exchange replaced by a $\sigma$ exchange.

The properties described above are, strictly speaking, only valid in the symmetric (massless) phase, where the $\left(2 N^{2}\right) \sigma$ and $\pi$ bosons are degenerate. However, in the broken phase, the properties are valid whenever momenta larger than the dynamical mass $\Sigma$ or $m_{\sigma}$ are considered, because then the degeneracy emerges too.

These properties also provide us with a general argument why the mean-field approach for four-fermion operators for Green functions corresponding to spin-zero channels (e.g., $\Gamma_{S}$ and $\Delta_{S}$ ) is reliable. For such channels planar contributions vanish and the next nonvanishing contributions (such as contributions containing crossed $\sigma$ and $\pi$ exchanges) are proportional to $1 / N$, thus small for large $N$. This implies that quantities such as the critical curve, dynamical mass, anomalous dimensions etc., are nearly independent of $N$, and are described rather well by the mean-field approach. To the contrary, the cancellation of scalars against pseudoscalars degrees of freedom does not occur in the vacuum polarization $\Pi$ which is a nonzero-spin channel.

\section{The fermion wave function}

The inclusion of relevant four-fermion interactions beyond the mean-field approach requires a reinvestigation of the $\mathrm{SDE}$ for the fermion wave function $\mathcal{Z}=Z_{2}$ (Eq. (90),

$$
S(p)=\frac{\mathcal{Z}\left(p^{2}\right)}{\hat{p}-\Sigma\left(p^{2}\right)},
$$

with $S$ the fermion propagator and $\Sigma$ the dynamical mass. In QED in the quenched-ladder approximation, the fermion wave function has a gauge dependent anomalous dimension. In the Landau gauge, this anomalous dimension vanishes and $\mathcal{Z}=1$.

We conjecture that the inclusion of relevant four-fermion interactions does not introduce an anomalous dimension for the fermion wave function other than already introduced by the gauge interactions. Thus, in the Landau gauge, the wave function $\mathcal{Z}$ is finite though it might deviate from unity. The main argument in support of the conjecture stated above is that only one full Yukawa vertex appears in the self-energy part, which means that anomalous dimensions of four-fermion interactions are not canceled. Only two fully dressed Yukawa vertices and a fully dressed scalar composite are RG invariant (anomalous dimensions cancel). Consequently a remnant power of the cutoff (related to anomalous dimension of a Yukawa vertex) lowers the degree of divergence of the self-energy part from a logarithmic divergence to a finite integral. Therefore, throughout this paper we assume $\mathcal{Z}=1$. 
A nice feature of the assumption that $\mathcal{Z}=1$ is that with the gauge interaction treated in the quenched-ladder approach the chiral and vector Ward-Takahashi identities (WTI's) are preserved, since in channels with spin-zero the planar $\sigma$ and $\pi$ exchanges cancel each other.

\section{SCALARS, PSEUDOSCALARS, AND CHARGE SCREENING}

Since, in the GNJL model, the scalars and pseudoscalars are neutral states which therefore do not couple to the photon field, their contribution to the vacuum polarization is described indirectly in terms of photon-fermion vertex corrections, and fermion self-energy corrections. Hence, in order to gain some intuition for the role of scalar degrees of freedom on the mechanism of charge screening, we analyze the two-loop contribution arising from $\sigma$ and $\pi$ exchanges to the vacuum polarization.

Let us consider an Abelian gauge-Higgs-Yukawa type of interaction described by the Lagrangian

$$
\begin{aligned}
\mathcal{L}_{G H Y} & =-\frac{1}{4} F_{\mu \nu} F^{\mu \nu}+\bar{\psi} i \gamma^{\mu} \partial_{\mu} \psi+\frac{1}{2}\left(\partial_{\mu} \sigma\right)^{2}+\frac{1}{2}\left(\partial_{\mu} \pi\right)^{2} \\
& -e_{0} \bar{\psi} \gamma^{\mu} A_{\mu} \psi-g_{Y} \bar{\psi}\left(\sigma+i \gamma_{5} \pi\right) \psi-V(\sigma, \pi)
\end{aligned}
$$

where the potential $V$ contains, e.g., mass terms, and a $\sigma^{4}$ type of interaction (i.e. a quartic scalar interaction). For simplicity, we ignore the effect of the potential $V$. In Appendix A, the two-loop contribution to $\Pi$ has been computed for the special case of $N=1$, see also Fig. 5. If the scalar and pseudoscalar fields in Eq. (50) are both in the adjoint representation of $U(N)$, the result, for arbitrary $N$, reads

$$
\Pi\left[q / \Lambda, \alpha_{0}, \lambda_{Y}\right] \approx \frac{N \alpha_{0}}{\pi}\left(\frac{2}{3}+\frac{\alpha_{0}}{2 \pi}-\frac{N \lambda_{Y}}{2 \pi}\right) \ln \frac{\Lambda}{q}+\left(\alpha_{0} / \pi\right) \mathcal{O}(1),
$$

with $\lambda_{Y}=g_{Y}^{2} / 4 \pi$. The $\beta$ function corresponding to such a vacuum polarization (Fig. 5) is

$$
\beta_{\alpha}\left(\alpha_{0}, \lambda_{Y}\right)=\frac{N \alpha_{0}^{2}}{\pi}\left(\frac{2}{3}+\frac{\alpha_{0}}{2 \pi}-\frac{N \lambda_{Y}}{2 \pi}\right) .
$$

The interesting result of this computation is difference in sign between terms corresponding to photon exchanges, and terms corresponding to (pseudo)scalar exchanges. From this point of view, the fundamental scalars and pseudoscalars in a gauge-Higgs-Yukawa system tend to decrease charge screening. Furthermore, we might be tempted to conclude that a nontrivial root of Eq. (52) could be realized whenever $N \lambda_{Y} / 2 \pi \sim 2 / 3$. However, the complete situation is more involved. The RG equation for, e.g., $\lambda_{Y}$ should be considered too, i.e., we should compute the $\beta$ functions of $\lambda_{Y}$, and of any quartic scalar coupling. If and only if a nontrivial (nonzero) UV stable fixed point for $\lambda_{Y}$ exists, the realization of a zero of Eq. (52) becomes a

\footnotetext{
${ }^{8}$ For a discussion of the renormalizability of non-Abelian gauge-Higgs-Yukawa models and nonAbelian GNJL models we refer to Refs. [55], and references therein.
} 
realistic option. In other words, such a scenario is only possible if the Yukawa interaction $\lambda_{Y}$ is nontrivial. The discussion in Sec. III implies that in order to obtain a nontrivial Yukawa coupling, the hyperscaling laws should be obeyed. The idea is that, nonperturbatively, close to the critical curve in the GNJL model, the scalar and pseudoscalar Yukawa interactions are nontrivial, and kinetic terms for the scalar and pseudoscalar composites are effectively induced via the appearance of a large anomalous dimension.

\section{THE VACUUM POLARIZATION IN THE 1/N EXPANSION}

The purpose of the present paper is to investigate the existence of an UV stable fixed point $\left(\alpha_{\star}, g_{\star}\right)$ of the gauge coupling, so that

$$
\beta_{\alpha}\left(\alpha_{\star}, g_{\star}\right)=0, \quad \eta_{\alpha} \equiv-\beta_{\alpha}^{\prime}\left(\alpha_{\star}, g_{\star}\right)>0,
$$

with $\eta_{\alpha}$ a critical index characterizing the RG flow in the neighborhood of $\left(\alpha_{\star}, g_{\star}\right)$. With $\alpha$ close to $\alpha_{\star}$ the $\beta$ function linearizes as

$$
\beta_{\alpha}\left(\alpha, g_{\star}\right) \approx \eta_{\alpha}\left(\alpha_{\star}-\alpha\right)
$$

The $\beta$ function for the gauge coupling follows from the $\mathrm{RG}$ transformation relating the charge $\alpha_{x}$ at scale $x$ to the charge $\alpha_{y}$ at scale $y$ via

$$
\alpha_{x}=\mathcal{R}_{\alpha}\left[x / y, \alpha_{y}, g_{y}\right]=\frac{\alpha_{y}}{1+\Pi\left[x / y, \alpha_{y}, g_{y}\right]}, \quad \Pi\left[1, \alpha_{x}, g_{x}\right]=0
$$

where $\Pi\left(x^{2}\right)=\Pi\left[x / y, \alpha_{y}, g_{y}\right]$ is the (Euclidean) vacuum polarization. The RG transformation should satisfy the RG semigroup property (with $x<y<z$ )

$$
\begin{aligned}
& \mathcal{R}_{\alpha}\left[x / y, \alpha_{y}, g_{y}\right]=\mathcal{R}_{\alpha}\left[x / z, \alpha_{z}, g_{z}\right]=\mathcal{R}_{\alpha}\left[x / y, \mathcal{R}_{\alpha}\left[y / z, \alpha_{z}, g_{z}\right], \mathcal{R}_{g}\left[y / z, \alpha_{z}, g_{z}\right]\right], \\
& \mathcal{R}_{\alpha}\left[x / y, \alpha_{\star}, g_{\star}\right]=\mathcal{R}_{\alpha}\left[x / z, \alpha_{\star}, g_{\star}\right],
\end{aligned}
$$

where $\mathcal{R}_{g}$ is the $\mathrm{RG}$ transformation for $g$, satisfying analogous equations. Then the $\beta$ function for $\alpha$ is defined as

$$
\left.\beta_{\alpha}\left(\alpha_{x}, g_{x}\right) \equiv \frac{\partial \mathcal{R}_{\alpha}\left[w, \alpha_{x}, g_{x}\right]}{\partial \ln w}\right|_{w=1} .
$$

The RG semigroup property (56) gives rise to the well known differential RG equation

$$
\beta_{\alpha}\left(\alpha_{y}, g_{y}\right)=y \frac{d \alpha_{y}}{d y}
$$

whose solution is

$$
\alpha_{x}=\sum_{n=0}^{\infty} \frac{1}{n !}\left(\ln \frac{x}{y}\right)^{n}\left[\beta_{\alpha}\left(\alpha_{y}, g_{y}\right) \frac{\partial}{\partial \alpha_{y}}\right]^{n} \alpha_{y} .
$$

To obtain a nontrivial theory in the IR the existence of an UV stable fixed point is required. Close to the UV stable fixed point $\left(\beta_{\alpha}=\beta_{g}=0\right)$, with $g_{y}=g_{\star}$, we have that 


$$
\left[\beta_{\alpha}\left(\alpha_{y}, g_{\star}\right) \frac{\partial}{\partial \alpha_{y}}\right]^{n} \alpha_{y} \approx \beta_{\alpha}\left(\alpha_{y}, g_{\star}\right)\left(\beta_{\alpha}^{\prime}\left(\alpha_{\star}, g_{\star}\right)\right)^{n-1}+\mathcal{O}\left(\beta^{2}\right)
$$

thus

$$
\alpha_{x} \approx \alpha_{\star}+\left(\alpha_{y}-\alpha_{\star}\right)\left(\frac{y}{x}\right)^{\eta_{\alpha}}
$$

which is the solution of Eq. (59) using Eq. (54). The above expressions are only valid if both $\alpha_{x}$ and $\alpha_{y}$ are in the neighborhood of the UV stable fixed point $\alpha_{\star}$, therefore we have the fine-tuning condition:

$$
\left|\frac{\left(\alpha_{y}-\alpha_{\star}\right)}{\alpha_{\star}}\left(\frac{y}{x}\right)^{\eta_{\alpha}}\right| \ll 1,
$$

which in case of $y \gg x$ implies that $\alpha_{y}$ is tuned sufficiently close to $\alpha_{\star}$.

In case of the Abelian GNJL model the gauge coupling $\alpha_{q}$ in the infrared (IR) region is related to the bare gauge coupling $\alpha_{0}$ via the RG transformation $\alpha_{q}=\mathcal{R}_{\alpha}\left[q / \Lambda, \alpha_{0}, g_{0}\right]$, where the four-fermion interactions contribute to the vacuum polarization $\Pi$ (Fig. 1) through the full photon fermion vertex, see Fig. 6. Assuming that the hyperscaling laws are satisfied, we can write the vacuum polarization for $\Lambda \gg q \geq\left|m_{\sigma}\right|$ (i.e., near $\left.g_{c}\left(\alpha_{0}\right)\right)$ as

$$
\Pi\left[q / \Lambda, \alpha_{0}, g_{0}\right]=f_{(1)}\left(\alpha_{0}, g_{0}\right) \ln \frac{\Lambda}{q}+f_{(2)}\left(\alpha_{0}, g_{0}\right)\left(\ln \frac{\Lambda}{q}\right)^{2}+f_{(3)}\left(\alpha_{0}, g_{0}\right)\left(\ln \frac{\Lambda}{q}\right)^{3}+\ldots
$$

where each factor of $\ln \Lambda / q$ corresponds to a single fermion loop with two outgoing photon lines. Thus the function $f_{(1)}$ represents the contribution of all diagrams to $\Pi$ in which the internal photon propagators are replaced by the bare or canonical form.

In order for $\Pi$ to give rise to a RG transformation satisfying Eqs. (55) and (56), it can be derived from Eq. (60) that the functions $f_{(1)}, f_{(2)}$, and $f_{(3)}$ should be related to $\beta_{\alpha}$ in the following way:

$$
\begin{aligned}
\alpha f_{(1)}(\alpha, g) & =\beta_{\alpha}(\alpha, g), \\
f_{(1)}^{2}-f_{(2)} & =\frac{1}{2 \alpha} \beta_{\alpha} \beta_{\alpha}^{\prime}, \\
f_{(1)}^{3}-2 f_{(1)} f_{(2)}+f_{(3)} & =\frac{1}{6 \alpha}\left[\beta_{\alpha} \frac{\partial}{\partial \alpha}\right]^{2} \beta_{\alpha}, \ldots
\end{aligned}
$$

These identities are nontrivial and require a high degree of self-consistency of the theory in the form of Ward identities.9 Since, within our approximation scheme, the Ward identities are respected, we assume that Eqs. (65)-(67) are satisfied. Equation (65) now relates the $\beta$ function to the function $f_{(1)}$. Clearly the $\beta$ function has a Gaussian or trivial fixed point at $\alpha_{0}=0$.

From this we also have that with $\alpha_{0} \rightarrow \alpha_{\star}$ at $g_{0}=g_{\star}$ (see Eqs. (54), (62), and (63)):

\footnotetext{
${ }^{9}$ The proof of Eqs. (65)-(67) can be performed order by order within perturbation theory.
} 


$$
\Pi\left[q / \Lambda, \alpha_{0}, g_{\star}\right] \approx \frac{\beta_{\alpha}\left(\alpha_{0}, g_{\star}\right)}{\alpha_{\star} \eta_{\alpha}}\left[-1+\left(\frac{\Lambda}{q}\right)^{\eta_{\alpha}}\right], \quad \frac{\beta_{\alpha}\left(\alpha_{0}, g_{\star}\right)}{\alpha_{\star} \eta_{\alpha}}\left[-1+\left(\frac{\Lambda}{q}\right)^{\eta_{\alpha}}\right] \ll 1 .
$$

Hence, $\Delta(q)$ of Eq. (142) is

$$
\Delta(q) \approx \frac{1}{q^{2}}\left\{1-\frac{\beta_{\alpha}\left(\alpha_{0}, g_{\star}\right)}{\alpha_{\star} \eta_{\alpha}}\left[-1+\left(\frac{\Lambda}{q}\right)^{\eta_{\alpha}}\right]\right\} .
$$

The second term on the right-hand side of Eq. (69) will only contribute via internal photon propagators to the functions $f_{(2)}, f_{(3)}$, etc. and not to the function $f_{(1)}$. In order to find an UV stable fixed point of $\beta_{\alpha}$, we only have to compute $f_{(1)}$ and, therefore, we neglect all corrections to $\Delta(q)$ other than canonical in internal photon propagators. Because the contribution of $\Pi$ is neglected, this procedure is identical to quenching the internal photon propagators, although it is not quenched in the sense of taking $N \rightarrow 0$ !

Now in correspondence with Eq. (18) we search for UV stable roots of Eq. (65), i.e., $f_{(1)}\left(\alpha_{0}, g_{c}\left(\alpha_{0}\right)\right)=0$. In case of pure QED (without four-fermion coupling) the function $f_{(1)}$ has been studied thoroughly by Johnson et al. in Refs. [56 58] and by Adler [59] in the context of massless QED. In Ref. [56], an expression for $f_{(1)}$ is obtained in term of the BS kernel $K^{(2)}$ (Sec. [V) as the single unknown Green function.

We mention that, although the strong belief of Johnson et al. in the possible existence of finite QED seems poorly motivated from the point of view of Wilson's RG methods [5] 10 their methods and techniques are sound and directly applicable to the GNJL model.

In Appendix B, we expose a brief derivation of the result of Ref. [56] and point out the applicability to the Abelian GNJL model. Then, by taking into account also relevant four-fermion interactions at $g_{0}=g_{c}\left(\alpha_{0}\right)$ via the BS kernel, we can derive from Eqs. (B1) and (65) that

$$
\beta_{\alpha}\left(\alpha_{0}, g_{c}\right)=\frac{N \alpha_{0}^{2}}{\pi}\left[\frac{2}{3}+\frac{\phi_{1}+\phi_{2}\left(2+\phi_{2}\right)}{1-\phi_{1}}+\phi_{3}\right],
$$

where the functions $\phi_{1}, \phi_{2}$, and $\phi_{3}$ are defined as follows:

$$
\begin{aligned}
& \phi_{1} \equiv-\lim _{\Lambda \rightarrow \infty} \frac{i e_{0}^{2}}{48 N} \int_{\Lambda} \frac{d^{4} p}{(2 \pi)^{4}} \tilde{\operatorname{Tr}}\left[\frac{\left(\gamma^{\mu} \hat{p} \gamma^{\alpha}-\gamma^{\alpha} \hat{p} \gamma^{\mu}\right)}{2 p^{4}} K^{(2)}(p, k)\left(\gamma_{\mu} \hat{k} \gamma_{\alpha}-\gamma_{\alpha} \hat{k} \gamma_{\mu}\right)\right], \\
& \phi_{2} \equiv-\lim _{\Lambda \rightarrow \infty} \frac{i e_{0}^{2}}{48 N} \int_{\Lambda} \frac{d^{4} p}{(2 \pi)^{4}} \tilde{\operatorname{Tr}}\left[\frac{\hat{p} \gamma^{\mu} \hat{p}}{p^{4}} K^{(2) \alpha}(p, k)\left(\gamma_{\mu} \hat{k} \gamma_{\alpha}-\gamma_{\alpha} \hat{k} \gamma_{\mu}\right)\right], \\
& \phi_{3} \equiv \lim _{\Lambda \rightarrow \infty} \frac{i e_{0}^{2}}{48 N} \int_{\Lambda} \frac{d^{4} p}{(2 \pi)^{4}} \tilde{\operatorname{Tr}}\left[\frac{\hat{p} \gamma^{\mu} \hat{p}}{p^{4}} K_{\alpha}^{(2) \alpha}(p, k) \hat{k} \gamma_{\mu} \hat{k}\right],
\end{aligned}
$$

with

\footnotetext{
10 The authors of [56] do not address the dynamical origin of the singular critical behavior (e.g., $\mathrm{D} \chi \mathrm{SB})$, which would be required for the realization of an UV stable fixed point in QED.
} 


$$
\begin{aligned}
K^{(2)}(p, k) & \left.\equiv K^{(2)}(p, p+q, k+q)\right|_{q=0}, \\
K^{(2) \alpha}(p, k) & \left.\equiv \frac{\partial}{\partial q_{\alpha}} K^{(2)}(p, p+q, k+q)\right|_{q=0}, \\
K_{\alpha}^{(2) \alpha}(p, k) & \left.\equiv \frac{\partial^{2}}{\partial q^{\alpha} \partial q_{\alpha}} K^{(2)}(p, p+q, k+q)\right|_{q=0} .
\end{aligned}
$$

The trace over spinor and flavor indices is defined as

$$
\tilde{\operatorname{Tr}}[L(p) K(p, k) R(k)] \equiv L_{d c}(p) K_{\substack{c d, a b \\ i i, j j}}(p, k) R_{b a}(k),
$$

with $L$ and $R$ some projectors and with appropriate summation over double spinor (4) and flavor indices $(\mathrm{N})$.

The BS kernels in Eqs. (71)-(73) contain, in principle, all diagrams except those corresponding to vacuum polarization corrections, since all internal photon propagators are canonical or "quenched." As was pointed out in Sec. IV, the $1 / N$ expansion states that the planar diagrams for the $\sigma$ and $\pi$ exchanges are dominant. The approximation for the BS kernel $K^{(2)}$, which generates the entire set of planar scalar and pseudoscalar skeleton diagrams including ladder photon exchanges for the vacuum polarization is the following: the BS kernel $K^{(2)}$ is approximated by its "lowest" order skeleton graph, i.e.,

$$
\begin{aligned}
K_{c c l, a b}^{(2)}(p, p+q, k+q) & =\frac{\delta_{i j} \delta_{k l}}{e_{0}^{2}}\left[\Gamma_{S c b}(k+q, p+q) \Delta_{S}(k-p) \Gamma_{S a d}(p, k)\right. \\
& \left.+\Gamma_{P c b}(k+q, p+q) \Delta_{P}(k-p) \Gamma_{P a d}(p, k)\right] \\
& +\delta_{i l} \delta_{k j} \gamma_{a d}^{\mu} \gamma_{c b}^{\nu} D_{\mu \nu}(k-p) .
\end{aligned}
$$

In the symmetric phase, the decomposition of the scalar Yukawa vertex $\Gamma_{S}$ is given by Eq. (21). Furthermore, due to chiral symmetry, we have the identities

$$
\left(i \gamma_{5}\right) \Gamma_{S}(k, p)=\Gamma_{P}(k, p), \quad \Delta_{S}(q)=\Delta_{P}(q),
$$

the $\sigma$ and $\pi$ propagators are degenerate. Second, it was shown in Ref. [47] that the structure function $F_{2}$ is rather small compared to the leading structure function $F_{1}$ (it is assumed that $F_{1}$ describes the leading asymptotic behavior of the Yukawa vertices). Therefore, we neglect contributions related to the scalar structure function $F_{2}$. Although it might be possible that the contribution coming from gauge interactions is smaller than corrections resulting from this structure function $F_{2}$, we keep the gauge interaction in order to compare with results mentioned in the literature. Thus, we take for $K^{(2)}$

$$
\begin{aligned}
K_{c d, a b}^{(2)}(p, p+q, k+q) & \approx \frac{\delta_{i j} \delta_{k l}}{e_{0}^{2}} F_{1}(k+q, p+q) F_{1}(p, k) \Delta_{S}(k-p)\left[\mathbf{1}_{a d} \mathbf{1}_{c b}+i \gamma_{5 a d} i \gamma_{5 c b}\right] \\
& +\delta_{i l} \delta_{k j} D_{\mu \nu}(k-p) \gamma_{a d}^{\mu} \gamma_{c b}^{\nu} .
\end{aligned}
$$

With this truncation for the BS kernel $K^{(2)}$, we can actually compute the $\phi_{j}$ functions (71)-(73), and subsequently analyze the $\beta$ function (70). The truncation (80) generates an infinite series of planar contributions to the vacuum polarization as the leading order in $1 / N$, see Fig. 7. As was discussed in Sec. IVB and shown in Appendix A up to two loops, the scalars and pseudoscalars give the same contribution in the functions $\phi_{j}$. The trace over flavor indices yields an overall factor of $N$ in the expressions for $\phi_{j}$ for contributions corresponding to $\sigma$ and $\pi$ exchanges. 


\section{COMPUTATION OF THE $\beta$ FUNCTION}

In computing the functions $\phi_{1}, \phi_{2}$, and $\phi_{3}$, we initially neglect the ladder photon exchange given in Eq. (80). Since such contributions were already computed in Ref. [56], it will be rather easy to include them later in the analysis.

It is straightforward to show that $\phi_{2}$ vanishes, within the proposed approximations. Using Eq. (80), we obtain from Eqs. (72) and (75) that

$$
\operatorname{Tr}\left[\hat{p} \gamma^{\mu} \hat{p} K^{(2) \alpha}(p, k)\left(\gamma_{\mu} \hat{k} \gamma_{\alpha}-\gamma_{\alpha} \hat{k} \gamma_{\mu}\right)\right] \propto \operatorname{Tr}\left[\hat{p} \gamma^{\mu} \hat{p}\left(\gamma_{\mu} \hat{k} \gamma_{\alpha}-\gamma_{\alpha} \hat{k} \gamma_{\mu}\right)\right]=0 .
$$

Thus $\phi_{2}\left(\alpha_{0}, g_{c}\right)=0$.

With Eq. (80), Eq. (71) for $\phi_{1}$ reads

$$
\begin{aligned}
\phi_{1}\left(\alpha_{0}, g_{c}\right)= & -\lim _{\Lambda \rightarrow \infty} \frac{i}{48 N} \int_{\Lambda} \frac{d^{4} p}{(2 \pi)^{4}} \frac{1}{2 p^{4}} \sum_{i=1}^{N} \sum_{j=1}^{N} \delta_{i i} \delta_{j j} F_{1}(k, p) F_{1}(p, k) \Delta_{S}(k-p) \\
& \times\left\{\operatorname{Tr}\left[\left(\gamma^{\mu} \hat{p} \gamma^{\alpha}-\gamma^{\alpha} \hat{p} \gamma^{\mu}\right)\left(\gamma_{\mu} \hat{k} \gamma_{\alpha}-\gamma_{\alpha} \hat{k} \gamma_{\mu}\right)\right]\right. \\
& \left.+\operatorname{Tr}\left[\left(\gamma^{\mu} \hat{p} \gamma^{\alpha}-\gamma^{\alpha} \hat{p} \gamma^{\mu}\right) i \gamma_{5}\left(\gamma_{\mu} \hat{k} \gamma_{\alpha}-\gamma_{\alpha} \hat{k} \gamma_{\mu}\right) i \gamma_{5}\right]\right\} \\
= & \lim _{\Lambda \rightarrow \infty} 2 N i \int_{\Lambda} \frac{d^{4} p}{(2 \pi)^{4}} \frac{(p+k) \cdot k}{(p+k)^{4}}\left[F_{1}(k+p, k)\right]^{2} \Delta_{S}(p),
\end{aligned}
$$

where we have performed a "harmless" symmetric in the fermion momenta, $F_{1}(p, k)=F_{1}(k, p)$, because of $C$-PT invariance. The overall factor $N$ results from tracing the flavor Kronecker $\delta$ functions, which is equivalent to closing index lines, see Sec. IVB. After a Wick rotation

$$
\phi_{1}\left(\alpha_{0}, g_{c}\right)=\lim _{\Lambda \rightarrow \infty} \frac{N}{8 \pi^{2}} \int_{0}^{\Lambda^{2}} d p^{2} \int \frac{d \Omega_{p}}{2 \pi^{2}} \frac{p^{2}\left(p \cdot k+k^{2}\right)}{(p+k)^{4}}\left[F_{1}(k+p, k)\right]^{2} \Delta_{S}(p) .
$$

Since the integrals for the functions $\phi_{j}$ are finite, we can write

$$
\phi_{1}\left(\alpha_{0}, g_{c}\right)=-N \int_{0}^{\infty} d u G_{1}(u)
$$

where $u=p^{2} / k^{2}$, and

$$
G_{1}\left(p^{2} / k^{2}\right) \equiv-\lim _{\Lambda \rightarrow \infty} \frac{1}{8 \pi^{2}} \int \frac{d \Omega_{p}}{2 \pi^{2}} \frac{k^{2} p^{2}\left(k \cdot p+k^{2}\right)}{(k+p)^{4}}\left[F_{1}(k+p, k)\right]^{2} \Delta_{S}(p) .
$$

The function $G_{1}$ is defined with a minus sign to make it a positive function, as will be shown to be the case later. The angular integral can be performed if we make use of the following Chebyshev expansion:

\footnotetext{
${ }^{11}$ The integral is finite, therefore translationally invariant.
} 


$$
\frac{k^{2}\left(k \cdot p+k^{2}\right)}{(k+p)^{4}}=\sum_{n=0}^{\infty} c_{n}\left(k^{2}, p^{2}\right) U_{n}(\cos \alpha), \quad \cos \alpha=\frac{k \cdot p}{k p},
$$

where

$$
\begin{aligned}
& c_{n}\left(k^{2}, p^{2}\right)=\frac{2}{\pi} \int_{0}^{\pi} d \alpha \sin ^{2} \alpha U_{n}(\cos \alpha) \frac{k^{2}\left(k \cdot p+k^{2}\right)}{(k+p)^{4}} \\
& c_{n}\left(k^{2}, p^{2}\right)=\frac{(-1)^{n}}{2}\left[(2+n) \theta\left(k^{2}-p^{2}\right)\left(\frac{p}{k}\right)^{n}-n \theta\left(p^{2}-k^{2}\right)\left(\frac{k}{p}\right)^{n+2}\right] .
\end{aligned}
$$

The Chebyshev expansion for the function $F_{1}$ was already introduced in Eq. (22) (and [47]). Thus, following analogous derivations in the Appendix of Ref. [47, the function $G_{1}$ can be expressed as

$$
G_{1}\left(p^{2} / k^{2}\right)=-\lim _{\Lambda \rightarrow \infty} \frac{p^{2} \Delta_{S}(p)}{8 \pi^{2}} \sum_{l, m, n=0}^{\infty} C_{l m n} c_{l}\left(k^{2}, p^{2}\right) f_{m}\left(k^{2}, p^{2}\right) f_{n}\left(k^{2}, p^{2}\right),
$$

where the fully symmetric index $C_{l m n}=1$, if $l+m+n=$ even and a triangle with sides $l$, $m, n$ exists, i.e., $|l-m| \leq n \leq l+m$. Otherwise $C_{l m n}=0$.

We approximate $G_{1}$ by keeping only the lowest order term in the Chebyshev expansion,

$$
G_{1}\left(p^{2} / k^{2}\right) \approx-\lim _{\Lambda \rightarrow \infty} \frac{p^{2} \Delta_{S}(p)}{8 \pi^{2}} c_{0}\left(k^{2}, p^{2}\right)\left[f_{0}\left(k^{2}, p^{2}\right)\right]^{2}
$$

where $f_{0}$ is decomposed into the two channel functions $F_{\mathrm{UV}}$ and $F_{\mathrm{IR}}$, see Eq. (24). Then, the asymptotics, $k^{2} \gg p^{2}$, respectively, $p^{2} \gg k^{2}$, of $G_{1}$ are well approximated by the lowest order Chebyshev term (90). Again, this is the two channel approximation for the Yukawa vertices [47. However, for momenta $k^{2} \sim p^{2}$ the channel approximation is not necessarily valid. So, how about $G_{1}(1)$ ? Since, from the appendix of Refs. 477, it follows that the Chebyshev coefficients

$$
f_{2 n}\left(k^{2}, p^{2}\right) \geq 0, \quad f_{2 n+1}\left(k^{2}, p^{2}\right) \leq 0,
$$

and from Eq. (88) that

$$
c_{n}\left(k^{2}, k^{2}\right)=\frac{(-1)^{n}}{2} \quad \longrightarrow \quad c_{2 n}\left(k^{2}, k^{2}\right) \geq 0, \quad c_{2 n+1}\left(k^{2}, k^{2}\right) \leq 0 .
$$

Hence, by taking into account the properties of $C_{l m n}$, we conclude that all terms of the series

$$
\sum_{l, m, n=0}^{\infty} C_{l m n} c_{l}\left(k^{2}, k^{2}\right) f_{m}\left(k^{2}, k^{2}\right) f_{n}\left(k^{2}, k^{2}\right)
$$

of $G_{1}$ are positive, and the lowest order term gives a lower bound on the series,

$$
c_{0}\left(k^{2}, k^{2}\right)\left[f_{0}\left(k^{2}, k^{2}\right)\right]^{2} \leq \sum_{l, m, n=0}^{\infty} C_{l m n} c_{l}\left(k^{2}, k^{2}\right) f_{m}\left(k^{2}, k^{2}\right) f_{n}\left(k^{2}, k^{2}\right) .
$$


Therefore, the approximation Eq. (90) is reliable for the asymptotics $k^{2} \gg p^{2}$, and $p^{2} \gg k^{2}$. Moreover, Eq. (90) is a lower bound on Eq. (89) at $k^{2}=p^{2}$, so that at least we will not overestimate the contribution of scalar and pseudoscalar composites to the vacuum polarization.

The function $G_{1}$ can now be computed, since $f_{0}$ is expressed in terms of the channel functions $F_{\mathrm{UV}}$ and $F_{\mathrm{IR}}$ of Eq. (24). Furthermore, from Eq. (88) we see that

$$
c_{0}\left(k^{2}, p^{2}\right)=\theta\left(k^{2}-p^{2}\right),
$$

and the only nonzero contribution to $G_{1}$ of Eq. (90) comes from the momenta $k^{2} \geq p^{2}$. Thus, using Eqs. (90), (24), and (95), we find

$$
G_{1}\left(p^{2} / k^{2}\right) \approx-\lim _{\Lambda \rightarrow \infty} \frac{p^{2} \Delta_{S}(p)}{8 \pi^{2}}\left[F_{\mathrm{UV}}\left(k^{2}, p^{2}\right)\right]^{2} \theta\left(k^{2}-p^{2}\right) .
$$

As was discussed in Sec. III, the ultraviolet channel function $F_{\mathrm{UV}}\left(k^{2}, p^{2}\right)$ is proportional to $(\Lambda / p)^{\eta / 2}$ and $p^{2} \Delta_{S}(p)$ is proportional to $(\Lambda / p)^{-\eta}$. Therefore, the cutoff dependence cancels in Eq. (96) as was expected and the angular integral Eq. (85) can indeed be written in terms of a function which depends only on the ratio of $p^{2} / k^{2}$.

The scaling form $\left(p^{2} \ll \Lambda^{2}\right)$ for the scalar propagator $\Delta_{S}(p)$ at $g_{0}=g_{c}\left(m_{\sigma}=0\right)$ is given by Eq. (26) and Eq. (28). The scaling form for the channel function $F_{\mathrm{UV}}\left(k^{2}, p^{2}\right)$ is given by Eqs. (34) and (36), with $p^{2} \leq k^{2} \ll \Lambda^{2}$. Inserting Eqs. (26) (with $m_{\sigma}=0$ ), (34), and (36) in Eq. (96), we obtain for $G_{1}$

$$
\begin{aligned}
G_{1}(u) & =\frac{\Gamma(2-\omega) \Gamma(2+\omega)}{8 \omega \gamma(\omega) \gamma(-\omega)} \\
& \times u\left[\gamma(\omega) I_{-\omega}\left(\sqrt{2 \lambda_{0} u}\right)-\gamma(-\omega) I_{\omega}\left(\sqrt{2 \lambda_{0} u}\right)\right]^{2} \theta(1-u),
\end{aligned}
$$

where $u=p^{2} / k^{2}$. Thus Eq. (84) is

$$
\begin{aligned}
\phi_{1}\left(\alpha_{0}, g_{c}\right) & \approx-N \zeta_{1}\left(\alpha_{0}\right), \\
\zeta_{1}\left(\alpha_{0}\right) & =\int_{0}^{1} d u G_{1}(u) \geq 0 .
\end{aligned}
$$

The function $G_{1}$ is positive, hence $\phi_{1}$ is negative. The integral over the function $G_{1}$ can be done explicitly by making use of the integral identity 2.15.19.1 in Volume 2 of Prudnikov et al. [60]. The result is

$$
\begin{aligned}
\zeta_{1}\left(\alpha_{0}\right) & =\frac{1}{\omega} \frac{\lambda_{0}}{2}\left\{\frac{1}{(2+\omega)} \frac{\Gamma(1-\omega) \gamma(-\omega)}{\Gamma(1+\omega) \gamma(\omega)}\left(\frac{\lambda_{0}}{2}\right)^{\omega}\right. \\
& \times{ }_{2} F_{3}\left(2+\omega, 1 / 2+\omega ; 3+\omega, 1+2 \omega, 1+\omega ; 2 \lambda_{0}\right) \\
& -{ }_{2} F_{3}\left(2,1 / 2 ; 3,1+\omega, 1-\omega ; 2 \lambda_{0}\right) \\
& +\frac{1}{(2-\omega)} \frac{\Gamma(1+\omega) \gamma(\omega)}{\Gamma(1-\omega) \gamma(-\omega)}\left(\frac{\lambda_{0}}{2}\right)^{-\omega} \\
& \left.\times{ }_{2} F_{3}\left(2-\omega, 1 / 2-\omega ; 3-\omega, 1-2 \omega, 1-\omega ; 2 \lambda_{0}\right)\right\} .
\end{aligned}
$$


The above analysis of the function $\phi_{1}$ is repeated for the function $\phi_{3}$. The second derivative, $K_{\alpha}^{(2) \alpha}(p, k)$ (see Eq. (76)), of the BS kernel given by Eq. (80) is

$$
K_{\alpha}^{(2) \alpha}(p, k) \propto \lim _{q \rightarrow 0} \frac{\partial^{2}}{\partial q^{\alpha} \partial q_{\alpha}} F_{1}(k+q, p+q) .
$$

The SDE for $F_{1}$ (in quenched-ladder approximation, see Fig. 3) is given by

$$
F_{1}(k+q, p+q)=1-i \lambda_{0} \int_{\Lambda} \frac{d^{4} r}{\pi^{2}} \frac{\left(r^{2}+(k-p) \cdot r\right)}{r^{2}(r+k-p)^{2}(r-p-q)^{2}} F_{1}(r+k-p, r),
$$

where we recall that we neglect the vertex function $F_{2}$. Thus

$$
\begin{aligned}
\lim _{q \rightarrow 0} \frac{\partial^{2}}{\partial q^{\alpha} \partial q_{\alpha}} F_{1}(k+q, p+q) & =-i \lambda_{0} \int_{\Lambda} \frac{d^{4} r}{\pi^{2}} \frac{\left(r^{2}+(k-p) \cdot r\right)}{r^{2}(r+k-p)^{2}} F_{1}(r+k-p, r) \\
& \times \lim _{q \rightarrow 0} \frac{\partial^{2}}{\partial q^{\alpha} \partial q_{\alpha}} \frac{1}{(r-p-q)^{2}} .
\end{aligned}
$$

By making use of the identity

$$
\frac{\partial}{\partial q^{\alpha}} \frac{\partial}{\partial q_{\alpha}} \frac{1}{q^{2}}=-4 \pi^{2} i \delta^{4}(q)
$$

we obtain

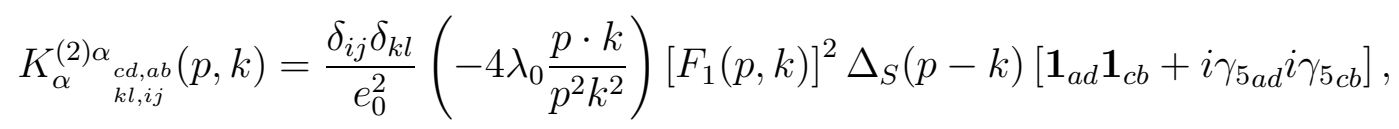

in Minkowsky formulation. Inserting the above expression in Eq. (73) the equation for $\phi_{3}$ takes the form (in Euclidean formulation)

$$
\phi_{3}\left(\alpha_{0}, g_{c}\right)=-\lim _{\Lambda \rightarrow \infty} \frac{\alpha_{0}}{\pi} \frac{N}{8 \pi^{2}} \int_{0}^{\Lambda^{2}} d p^{2} \int \frac{d \Omega_{p}}{2 \pi^{2}} \frac{p^{2}}{k^{2}} \frac{\left(p \cdot k+k^{2}\right)^{3}}{(p+k)^{6}}\left[F_{1}(k+p, k)\right]^{2} \Delta_{S}(p) .
$$

Then

$$
\begin{aligned}
\phi_{3}\left(\alpha_{0}, g_{c}\right) & =N \int_{0}^{\infty} d u G_{3}(u), \\
G_{3}\left(p^{2} / k^{2}\right) & \equiv \lim _{\Lambda \rightarrow \infty}-\frac{\alpha_{0}}{\pi} \frac{1}{8 \pi^{2}} \int \frac{d \Omega_{p}}{2 \pi^{2}} \frac{p^{2}\left(k \cdot p+k^{2}\right)^{3}}{(k+p)^{6}}\left[F_{1}(k+p, k)\right]^{2} \Delta_{S}(p) .
\end{aligned}
$$

We use the following Chebyshev expansion:

$$
\frac{\left(k \cdot p+k^{2}\right)^{3}}{(k+p)^{6}}=\sum_{n=0}^{\infty} d_{n}\left(k^{2}, p^{2}\right) U_{n}(\cos \alpha), \quad \cos \alpha=\frac{k \cdot p}{k p},
$$

where 


$$
\begin{aligned}
d_{n}\left(k^{2}, p^{2}\right) & =\frac{2}{\pi} \int_{0}^{\pi} d \alpha \sin ^{2} \alpha U_{n}(\cos \alpha) \frac{\left(p \cdot k+k^{2}\right)^{3}}{(p+k)^{6}} \\
d_{0}\left(k^{2}, p^{2}\right) & =\left(1-\frac{3 p^{2}}{4 k^{2}}\right) \theta\left(k^{2}-p^{2}\right) \\
d_{n}\left(k^{2}, p^{2}\right) & =\frac{(-1)^{n}}{8}\left\{n+1+\left[6+\sum_{l=0}^{n-1}(4+l)\right]\left(1-\frac{p^{2}}{k^{2}}\right)\right\} \theta\left(k^{2}-p^{2}\right)\left(\frac{p}{k}\right)^{n} \\
& -\frac{(-1)^{n}}{8}\left\{n+1-\left[\sum_{l=0}^{n-1}(2-l)\right]\left(1-\frac{k^{2}}{p^{2}}\right)\right\} \theta\left(p^{2}-k^{2}\right)\left(\frac{k}{p}\right)^{n}, \quad n \geq 1 .
\end{aligned}
$$

The function $G_{3}$ can be expressed as

$$
G_{3}\left(p^{2} / k^{2}\right)=\lim _{\Lambda \rightarrow \infty}-\frac{\alpha_{0}}{\pi} \frac{p^{2} \Delta_{S}(p)}{8 \pi^{2}} \sum_{l, m, n=0}^{\infty} C_{l m n} d_{l}\left(k^{2}, p^{2}\right) f_{m}\left(k^{2}, p^{2}\right) f_{n}\left(k^{2}, p^{2}\right) .
$$

We also approximate $G_{3}$ by keeping only the lowest order term in the Chebyshev expansion,

$$
G_{3}\left(p^{2} / k^{2}\right) \approx \lim _{\Lambda \rightarrow \infty}-\frac{\alpha_{0}}{\pi} \frac{p^{2} \Delta_{S}(p)}{8 \pi^{2}} d_{0}\left(k^{2}, p^{2}\right)\left[f_{0}\left(k^{2}, p^{2}\right)\right]^{2}
$$

Then, again the asymptotics, $k^{2} \gg p^{2}$, respectively, $p^{2} \gg k^{2}$, of $G_{3}$ are well approximated by the lowest order Chebyshev term. Moreover, for momenta $k^{2}=p^{2}$ the approximation Eq. (114) is exact, since $d_{n}\left(k^{2}, k^{2}\right)=0$ for all $n \geq 1$. Therefore, the approximation Eq. (114) is even better than the analogous approximation, Eq. (90), to $G_{1}$. Furthermore, from Eq. (111) we see that the only nonzero contributions to $G_{3}$ of Eq. (114) are given by momenta $k^{2} \geq p^{2}$. Thus, using Eqs. (114), (24), and (111), we find

$$
G_{3}\left(p^{2} / k^{2}\right) \approx \lim _{\Lambda \rightarrow \infty}-\frac{\alpha_{0}}{\pi}\left(1-\frac{3 p^{2}}{4 k^{2}}\right) \frac{p^{2} \Delta_{S}(p)}{8 \pi^{2}}\left[F_{\mathrm{UV}}\left(k^{2}, p^{2}\right)\right]^{2} \theta\left(k^{2}-p^{2}\right) .
$$

Substituting Eqs. (26), (34), and (36) in Eq. (115), we obtain for $G_{3}$

$$
\begin{aligned}
G_{3}(u) & =\frac{\alpha_{0}}{\pi}\left(1-\frac{3 u}{4}\right) \frac{\Gamma(2-\omega) \Gamma(2+\omega)}{8 \omega \gamma(\omega) \gamma(-\omega)} \\
& \times u\left[\gamma(\omega) I_{-\omega}\left(\sqrt{2 \lambda_{0} u}\right)-\gamma(-\omega) I_{\omega}\left(\sqrt{2 \lambda_{0} u}\right)\right]^{2} \theta(1-u),
\end{aligned}
$$

where $u=p^{2} / k^{2}$. Thus Eq. (107) is

$$
\begin{aligned}
\phi_{3}\left(\alpha_{0}, g_{c}\right) & \approx N \zeta_{3}\left(\alpha_{0}\right), \\
\zeta_{3}\left(\alpha_{0}\right) & =\int_{0}^{1} d u G_{3}(u) \geq 0 .
\end{aligned}
$$

The function $\phi_{3}$ is positive, and can be computed in the same way as $\phi_{1}$. The result is

$$
\zeta_{3}\left(\alpha_{0}\right)=\frac{\alpha_{0}}{\pi}\left[\zeta_{1}\left(\alpha_{0}\right)-\tau\left(\alpha_{0}\right)\right],
$$


where

$$
\begin{aligned}
\tau\left(\alpha_{0}\right) & =\frac{3}{4 \omega} \frac{\lambda_{0}}{2}\left\{\frac{1}{(3+\omega)} \frac{\Gamma(1-\omega) \gamma(-\omega)}{\Gamma(1+\omega) \gamma(\omega)}\left(\frac{\lambda_{0}}{2}\right)^{\omega}\right. \\
& \times{ }_{2} F_{3}\left(3+\omega, 1 / 2+\omega ; 4+\omega, 1+2 \omega, 1+\omega ; 2 \lambda_{0}\right) \\
& -\frac{2}{3}{ }_{2} F_{3}\left(3,1 / 2 ; 4,1+\omega, 1-\omega ; 2 \lambda_{0}\right) \\
& +\frac{1}{(3-\omega)} \frac{\Gamma(1+\omega) \gamma(\omega)}{\Gamma(1-\omega) \gamma(-\omega)}\left(\frac{\lambda_{0}}{2}\right)^{-\omega} \\
& \left.\times{ }_{2} F_{3}\left(3-\omega, 1 / 2-\omega ; 4-\omega, 1-2 \omega, 1-\omega ; 2 \lambda_{0}\right)\right\} .
\end{aligned}
$$

In the computation of the functions $\phi_{1}, \phi_{2}$, and $\phi_{3}$ the ladder (planar) photon exchanges have been neglected. After reinstating the ladder photon exchange term of Eq. (80), we obtain, together with Eqs. (98) and (117), that

$$
\phi_{1}\left(\alpha_{0}, g_{c}\left(\alpha_{0}\right)\right)=\frac{\alpha_{0}}{2 \pi}-N \zeta_{1}\left(\alpha_{0}\right), \quad \phi_{2}\left(\alpha_{0}, g_{c}\left(\alpha_{0}\right)\right)=0, \quad \phi_{3}\left(\alpha_{0}, g_{c}\left(\alpha_{0}\right)\right)=N \zeta_{3}\left(\alpha_{0}\right) .
$$

The ladder photon exchange only contributes to $\phi_{1}$, see again [56]. After substitution of Eq. (121) in Eq. (70), the $\beta$ function reads

$$
\beta_{\alpha}\left(\alpha_{0}, g_{c}\right)=\frac{N \alpha_{0}^{2}}{\pi}\left[\frac{2}{3}+\frac{\alpha_{0} / 2 \pi-N \zeta_{1}\left(\alpha_{0}\right)}{1-\alpha_{0} / 2 \pi+N \zeta_{1}\left(\alpha_{0}\right)}+N \zeta_{3}\left(\alpha_{0}\right)\right],
$$

where explicit expressions for $\zeta_{1}$ and $\zeta_{3}$ are given by Eq. (100) and Eqs. (119) and (120).

\section{UV STABLE FIXED POINTS}

Let us start analyzing Eq. (122) by first considering the properties of the functions $\zeta_{1}\left(\alpha_{0}\right)$ and $\zeta_{3}\left(\alpha_{0}\right)$. For $\alpha_{0}$ small, the expansion of the functions $\zeta_{1}$ and $\zeta_{3}$ can be computed from Eqs. (100) and (119). The result is

$$
\zeta_{1}\left(\alpha_{0}\right) \approx \frac{3 \alpha_{0}}{2 \pi}+\mathcal{O}\left(\alpha_{0}^{2}\right), \quad \zeta_{3}\left(\alpha_{0}\right) \approx \frac{15}{16} \frac{\alpha_{0}^{2}}{\pi^{2}}+\mathcal{O}\left(\alpha_{0}^{3}\right)
$$

showing that $\zeta_{3}$ vanishes faster that $\zeta_{1}$ for $\alpha_{0} \rightarrow 0$. The functions $\zeta_{1}\left(\alpha_{0}\right)$ and $\zeta_{3}\left(\alpha_{0}\right)$ have been plotted versus $\alpha_{0} / \alpha_{c}$ in Fig. 8. First, it is clear that $\zeta_{1}$ and $\zeta_{3}$, are positive, and have a maximum at some intermediate value of $0<\alpha_{0}<\alpha_{c}=\pi / 3$. For instance, $\zeta_{1}$ has a maximum $\zeta_{1} \approx 0.123$ at $\alpha_{0} / \alpha_{c} \approx 0.58(\omega \approx 0.65)$. Second, the functions $\zeta_{1}$ and $\zeta_{3}$ vanish at the pure NJL point $\alpha_{0}=0$ in accordance with Eq. (123), and at the CPT point $\alpha_{0}=\alpha_{c}$. At $\alpha_{0}=0$, we can consider this is as a reflection of the fact that hyperscaling breaks down due to logarithmic corrections; the "effective" Yukawa coupling is trivial, therefore vanishes. At $\alpha_{0}=\alpha_{c}$, where the critical exponents become singular, the vanishing of $\zeta_{1}$ and $\zeta_{3}$ is related to the dynamics of the conformal phase transition $(\mathrm{CPT})$, which has been 
thoroughly discussed in Ref. [61]. There are no light $\sigma$ and $\pi$ exchanges in the symmetric phase 47] which consequently implies the absence of effective Yukawa interactions. एँ]

Let us compare the $\beta$ function (122) with the $\beta$ function (52) of the gauge-HiggsYukawa model (50) in the $1 / N$ expansion. Then, the entire set of planar $\sigma$ and $\pi$ exchanges is generated by the kernel

$$
K_{c d, a b}^{(2)}(p, p+q, k+q) \approx \delta_{i j} \delta_{k l} \frac{g_{Y}^{2}}{e_{0}^{2}} \Delta_{S}(k-p)\left[\mathbf{1}_{a d} \mathbf{1}_{c b}+i \gamma_{5 a d} i \gamma_{5_{c b}}\right]
$$

where $\Delta_{S}(p)=1 / p^{2}$. With such a kernel, $\phi_{2}$ and $\phi_{3}$ are zero, because the right-hand side of Eq. (124) does not depend on the momentum $q$. The expression for $\phi_{1}$, in this case, can be computed straightforwardly $\left(\lambda_{Y}=g_{Y}^{2} / 4 \pi\right)$;

$$
\phi_{1}=\lim _{\Lambda \rightarrow \infty} 2 N g_{Y}^{2} i \int_{\Lambda} \frac{d^{4} p}{(2 \pi)^{4}} \frac{p \cdot k}{p^{4}} \frac{1}{(p-k)^{2}}=-\frac{N \lambda_{Y}}{2 \pi} .
$$

Again we introduce the ladder photon exchanges by the replacement

$$
\phi_{1}\left(\lambda_{Y}\right) \quad \longrightarrow \quad \phi_{1}\left(\alpha_{0}, \lambda_{Y}\right)=\frac{\alpha_{0}}{2 \pi}-\frac{N \lambda_{Y}}{2 \pi} .
$$

Hence, in this case, the $\beta$ function is

$$
\beta_{\alpha}\left(\alpha_{0}, \lambda_{Y}\right)=\frac{N \alpha_{0}^{2}}{\pi}\left[\frac{2}{3}+\frac{\alpha_{0} / 2 \pi-N \lambda_{Y} / 2 \pi}{1-\alpha_{0} / 2 \pi+N \lambda_{Y} / 2 \pi}\right] .
$$

Comparing the $\beta$ functions (122) and (127) leads to the suggestion that $\zeta_{1}\left(\alpha_{0}\right)$ is analogous to the Yukawa coupling $\lambda_{Y}$ in a gauge-Higgs-Yukawa model, $\zeta_{1}\left(\alpha_{0}\right) \sim \lambda_{Y} / 2 \pi$.

This is a crucial point. The general consensus is that for a gauge-Higgs-Yukawa model the Yukawa interaction $\lambda_{Y}$ is trivial, thus $\lambda_{Y} \rightarrow 0$ in Eq. (127). However, the situation is essentially different for $\zeta_{1}$ in the GNJL model. There the "effective" coupling $\zeta_{1}$ is formed by the exchange of $\sigma$ and $\pi$ bosons, with the Yukawa vertices, and (pseudo)scalar propagators fully dressed (i.e., the skeleton expansion). The cancellation of the $Z$ factors, see Sec. ПII, which is related to the fact that the hyperscaling equations are satisfied, gives rise to a finite nonzero $\zeta_{1}\left(\alpha_{0}\right)$ at the critical curve $\left(g_{0}=g_{c}\right)$ for $0<\alpha_{0}<\alpha_{c}$. The other nonzero function $\zeta_{3}$ results from taking into account fully dressed Yukawa vertices.

Let us now the discuss the possible existence of UV stable fixed points. A necessary but not a sufficient condition for the realization of an UV stable fixed point is that $N \zeta_{1}$ has to be larger than both $N \zeta_{3}$ and $\alpha_{0} / 2 \pi$, and $N \zeta_{1} \sim \mathcal{O}(1)$. For large $N$, the contribution of the planar photon exchanges (represented by the $\alpha_{0} / 2 \pi$ terms) is negligible with respect to $N \zeta_{1}$ and $N \zeta_{3}$. Moreover Fig. 8 shows, for $\alpha_{0}$ small, that $\zeta_{1}$ is considerably larger than $\zeta_{3}$. This means that only for flavors $N$ larger than some critical value $N_{c}$ UV stable fixed points can be obtained.

\footnotetext{
${ }^{12}$ Moreover at the CPT point four-fermion interaction are marginal instead of relevant, and start to mix with the gauge interaction, hence the analysis becomes considerably more complicated.
} 
By substituting the expressions (100) and (119) for $\zeta_{1}$ and $\zeta_{3}$ in Eq. (122), we can straightforwardly analyze the $\beta$ function graphically. In Fig. 9 the $\beta$ function is plotted for various values of $N$. Figure 9 shows that for values of $N>N_{c}$, with $55>N_{c}>54$, UV stable fixed points exist, the largest being $\alpha_{\star} \approx 0.13$;

$$
\begin{array}{ll}
N=55: & \beta_{\alpha}\left(0.13, g_{c}(0.13)\right) \approx 0, \quad \eta_{\alpha}=-\beta_{\alpha}^{\prime}\left(0.13, g_{c}(0.13)\right) \approx 0.07 \\
N=60: & \beta_{\alpha}\left(0.1, g_{c}(0.1)\right) \approx 0, \quad \eta_{\alpha}=-\beta_{\alpha}^{\prime}\left(0.1, g_{c}(0.1)\right) \approx 0.15
\end{array}
$$

In accordance with Eqs. (15) and (53), the fixed points are first-order zeros of $\beta$. The general pattern is clear; the larger $N$, with $N>N_{c}$, the smaller will be the UV stable fixed point, but the larger will be the critical exponent $\eta_{\alpha}$.

The above pattern also suggests that when $N \rightarrow \infty$ the UV stable fixed point $\alpha_{\star} \rightarrow 0$ and we would obtain an asymptotically free theory. This is not the case. It was shown in Refs. [45,47] as $\alpha_{0}$ goes to zero that a logarithmic correction appears in the expression for the scalar propagator $\Delta_{S}$. In fact, the scaling form for $\Delta_{S}$ (with $q / \Lambda \ll 1$ ) is only valid for values of $\omega$ so that $\left(q^{2} / \Lambda^{2}\right)^{\omega} \gg q^{2} / \Lambda^{2}$, see Ref. [47]. The logarithmic correction gives rise to the breakdown of hyperscaling relations and is synonymous to triviality of the four-fermion interactions (the NJL model). Since our results rely heavily on the existence of scaling forms such as Eqs. (26) and (34), we can only trust our results for values of $\alpha_{\star}$ not too small.

Since we have made use of results obtained in the quenched approximation, we mention that the plots of the $\beta$ function are (at the most) reliable at or in the vicinity of the UV stable fixed points at which the quenched approach is self consistent, see Eq. (68).

In Fig. 10, the case of $N=60$ fermion flavors is compared with the one-loop $\beta$ function of QED. For very small values of $\alpha_{0}<1 / 100$ indeed the one-loop QED result coincides with that of the GNJL model, however for larger values of $\alpha_{0}$ the $\beta$ function (122) deviates from the one-loop expression, and eventually an UV stable fixed point is realized at $\alpha_{\star} \approx 0.1$.

The analysis shows that a rather large number of flavors,

$$
N>N_{c} \approx 54
$$

is required to obtain UV stable fixed points. From the point of view of the $1 / N$ expansion this seems a consistent result, since other than planar contributions are suppressed by at least factors of (say) $1 / N_{c}$. However, from the phenomenological point of view, the result is unsatisfactory, since it implies that the unquenched Abelian GNJL model (exhibiting UV stable fixed points) is only practically applicable for models which have at least $N_{c}$ fermion flavors (fractions rounded up). Therefore, it is appropriate to discuss how $N_{c}$ depends on the approximation.

First, we stress that the second term on the right-hand side in Eq. (122) containing the $\zeta_{1}$ function causes the suppression of charge screening and is responsible for the possible realization of an UV stable fixed point. The denominator in the second term is a direct consequence of the resummation of the infinite ladder $\sigma$ and $\pi$ exchanges, and it is mainly due to this denominator $1+N \zeta_{1}$ that the critical number of fermion flavors $N_{c}$ is large.

Second, the existence of an UV stable fixed point for a specific number of fermion flavors $N$ depends on the interplay between the functions $\zeta_{1}$ and $\zeta_{3}$, which are given in terms of integrals of the functions $G_{1}$ and $G_{3}$. Let us recall that the lowest order Chebyshev expansion for $G_{1}$, Eq. (90) is a lower bound on $G_{1}$ of Eq. (39), since all terms of the Chebyshev 
expansion are positive at $k^{2}=p^{2}$, the same cannot be said about the approximation (114) for $G_{3}$. Thus keeping more terms in the Chebyshev expansion leads to an increase of $\zeta_{1}$, whereas the effect on $\zeta_{3}$ is less clear, because of the alternating Chebyshev series for $\zeta_{3}$. Therefore, an improvement of the computation of $\zeta_{1}$ will probably lead to a decrease of the critical flavor number $N_{c}$.

Moreover, in the computation of the functions $\zeta_{1}$ and $\zeta_{3}$ we have used Yukawa vertices $\left(\Gamma_{S}\right)$ and $\sigma$ and $\pi$ propagators $\left(\Delta_{S}\right)$ which were obtained in the quenched-ladder approximation. An interesting question is whether the improvement of the ladder approximation for the gauge interaction (e.g., by including crossed photon exchanges) leads to a increase of $\zeta_{1}$, and thus a decrease of $N_{c}$.

Finally, we recall that we have neglected the effect of the Yukawa vertex function $F_{2}$ (Eq. (21)), but clearly the inclusion of $F_{2}$ in the analysis could change the results quantitatively. Whether such an improvement will tend to increase or decrease $N_{c}$ remains unclear at this stage.

\section{CONCLUSION}

There are strong indications that four-fermion interactions become relevant near the chiral phase transition in GNJL models in four dimensions, due to the appearance of a large anomalous dimension. The main objective of this paper was to study the effect of such relevant four-fermion interactions on the vacuum polarization of the gauge coupling and to reinvestigate the problem of triviality for a particular Abelian GNJL model with $N$ fermion flavors. To obtain new results, the four-fermion interactions had to be taken into account beyond the commonly used Hartree-Fock or mean-field approach.

The crucial feature of the GNJL model, within the quenched mean-field approximation, is that a nontrivial Yukawa interaction (i.e., an interaction between composite (pseudo)scalars and fermions) exists for $0<\alpha_{0}<\alpha_{c}$. The existence of such a nontrivial Yukawa interaction requires the cancellation of renormalization constants of the $\sigma$ and $\pi$ fields in fermionantifermion scattering amplitudes such as the BS kernel $K^{(2)}$. This is analogous to the requirement of hyperscaling (see Sec. [II]). If the hyperscaling equations are satisfied, then only two of the critical exponents are independent, e.g., $\eta$ and $\gamma$, see Eqs. (19) and (27). The existence of hyperscaling relations between the critical exponents is intimately connected with the existence of Ward-Takahashi identities (and thus the Goldstone mechanism) arising from the continuous symmetries of the model.

The skeleton expansion for the BS kernel $K^{(2)}$ provides a natural framework to take into account the anomalous dimensions of Yukawa vertices and $\sigma$ and $\pi$ propagators. Within the skeleton expansion, $\sigma$ and $\pi$ exchanges are described in terms of fully dressed Yukawa vertices and $\sigma$ and $\pi$ propagators. The actual computation of the anomalous dimension, and the resolution of the scaling form requires a solution of the SDE's for Yukawa vertices, and $\sigma$ and $\pi$ bosons.

In previous work such fully dressed Yukawa vertices and $\sigma$ and $\pi$ propagators have been analyzed in the quenched-ladder mean-field approximation, see Ref. [47] and references therein. To make use of these results consistently, we used the following approximations.

First, we assumed that the bare coupling parameters are fine-tuned close to the critical point, i.e., close to an UV stable fixed point, at which $\beta_{g} \approx 0$ and $\beta_{\alpha} \approx 0$. In that case, the 
quenched or canonical approximation for the photon propagator is self-consistent. Second, the gauge-interaction is considered in the ladder form, with bare vertices. Third, we used the $1 / N$ expansion (with $N$ the number of fermion flavors) which states that planar $\sigma$ and $\pi$ exchanges describe the leading contribution to Green functions for large $N$. Then, due to the specific form of the chiral symmetry with both scalars and pseudoscalar in the adjoint representation of the $U_{L}(N) \times U_{R}(N)$ symmetry, we argued that in so-called zero-spin channels (such as Yukawa vertices and $\sigma$ and $\pi$ propagators) the planar $\sigma$ and $\pi$ exchanges cancel each other for momenta larger than the mass of the $\sigma$ boson (in fact in the symmetric phase this cancellation is exact). Moreover, an important property of the planar (ladder) approximations is that they respect the vector and chiral Ward-Takahashi identities.

The method of Ref. [56], provides a nonperturbative framework independent of the fermion wave function $\mathcal{Z}$, and allowed us to compute the contributions of the infinite set of planar $\sigma$ and $\pi$ exchanges to the vacuum polarization. The result of the computations is that the GNJL model exhibits an UV stable fixed point, $\beta_{\alpha}\left(\alpha_{\star}, g_{c}\left(\alpha_{\star}\right)\right)=0$, for any value of $N$ that exceeds some critical value $N_{c}\left(N>N_{c}\right)$. This critical number of flavors turned out to be $N_{c} \approx 54$. The larger the number of fermion flavors, the smaller the UV stable fixed point $\alpha_{\star}$ will be, provided $N>N_{c}$ and $\alpha_{\star}$ not too small. Since our results are derived on the basis of the existence of hyperscaling laws, we cannot extrapolate our results into the region where hyperscaling breaks down due to logarithmic violations, i.e., when $\alpha_{\star} \rightarrow 0$ $(N \rightarrow \infty)$.

From a phenomenological point of view, the large value for $N_{c}$ puts questions to the applicability of the GNJL. However, we have given a few arguments in the previous section suggesting that $N_{c}$ could be rather sensitive to approximations, and that an improvement of the approximations and calculations will probably lead to a smaller value for $N_{c}$.

The realization of an UV stable fixed point is motivated by the observation that contributions of planar $\sigma$ and $\pi$ exchanges to the vacuum polarization, in an Abelian gauge-HiggsYukawa model, have identical sign, and tend to reduce screening. In analogy, four-fermion interactions describe attractive forces between virtual fermion-antifermion pairs in the vacuum polarization.

The conventional leading term in the vacuum polarization is the one-loop correction describing the creation of fermion-antifermion pairs. These virtual pairs can be considered as dipoles causing the screening; the vacuum is a medium of the insulator type. Such a screening is proportional to the coupling $\alpha_{0}$ and proportional to the number of fermion flavors $N$. However, if a particular fraction of the total amount of fermion-antifermion pairs created are correlated by attractive four-fermion interactions, represented by $\sigma$ and $\pi$ exchanges, then clearly these composite neutral states are not capable of screening. The negative term $N \zeta_{1}$ in the $\beta$ function (122) represents the contributions and the attractive nature of four-fermion interactions in the vacuum polarization.

Within the quenched-ladder mean-field approximation, the critical curve and critical exponents are independent of the number of fermion flavors. Within our approximation scheme, the mechanism of charge screening clearly is flavor dependent, since the total number of virtual fermion-antifermion pairs is proportional to $N$ and the total number of composite scalars and pseudoscalars grows as $2 N^{2}$. The larger the number of flavors, the stronger the effect of four-fermion interactions. The fixed point appears when the virtual pairs completely loose their ability to screen. 
The existence of an UV stable fixed point implies a nontrivial continuum limit of the Abelian GNJL model. The analysis presented here suggest that in the full unquenched GNJL model the critical line is replaced by an UV stable fixed point (on the critical line) whose exact positions depends on the number of fermion flavors. If the number of fermion flavors is below some specific value, the critical four-fermion dynamics are not sufficient to yield an UV stable fixed point. In that case the unquenched GNJL model only has a trivial (IR) fixed point and the chiral phase transition is of the mean-field type.

\section{ACKNOWLEDGMENTS}

The author wishes to thank Valery Gusynin for the enjoyable and fruitful collaboration, the numerous stimulating discussions, and important suggestions. It is a pleasure to thank Marinus Winnink for useful comments and encouragement.

\section{APPENDIX A: TWO-LOOP VACUUM POLARIZATION}

In this appendix we compute two-loop vacuum polarization corrections including $\sigma$ and $\pi$ exchanges, see Fig. 5. We derive the two-loop contribution by making use of the one-loop computation of the photon-fermion vertex [62,63].

The SDE for vacuum polarization tensor reads $(N=1)$

$$
\Pi^{\mu \nu}\left(q^{2}\right)=i e_{0}^{2} \int_{\Lambda} \frac{d^{4} k}{(2 \pi)^{4}} \operatorname{Tr}\left[\gamma^{\mu} S(k+q) \Gamma^{\nu}(k+q, k) S(k)\right] .
$$

Assuming that the WTI's are respected, the vacuum polarization tensor is transverse: $\Pi^{\mu \nu}(q)=\left(-g^{\mu \nu} q^{2}+q^{\mu} q^{\nu}\right) \Pi\left(q^{2}\right)$, so that

$$
\Pi\left(q^{2}\right)=-\frac{i e_{0}^{2}}{3 q^{2}} \int_{\Lambda} \frac{d^{4} k}{(2 \pi)^{4}} \operatorname{Tr}\left[\gamma_{\mu} S(k+q) \Gamma^{\mu}(k+q, k) S(k)\right] .
$$

Let us write and denote the one-loop vertex and self-energy corrections with a subscript (1) as follows:

$$
\Gamma^{\mu}(k, p)=\gamma^{\mu}+\Lambda_{(1)}^{\mu}(k, p), \quad S(p)=\frac{\hat{p}}{p^{2}}\left[1+\mathcal{Z}_{(1)}\left(p^{2}\right)\right] .
$$

Besides a photon exchange, we take into account a scalar and pseudoscalar exchange in the one-loop vertex, and self-energy, i.e.,

$$
\begin{aligned}
& \Lambda_{(1)}^{\mu}(k, p)=\Lambda_{(1 V)}^{\mu}(k, p)+\Lambda_{(1 S)}^{\mu}(k, p)+\Lambda_{(1 P)}^{\mu}(k, p), \\
& \hat{p} \mathcal{Z}_{(1)}\left(p^{2}\right)=-\Sigma_{(1 V)}(p)-\Sigma_{(1 S)}(p)-\Sigma_{(1 P)}(p) .
\end{aligned}
$$

With one-loop vertex corrections 


$$
\begin{aligned}
\left(-i e_{0}\right) \Lambda_{(1 V)}^{\mu}(k, p)= & \int_{\Lambda} \frac{d^{4} w}{(2 \pi)^{4}}\left(-i e_{0}\right) \gamma^{\lambda} i S(k-w) \\
& \times\left(-i e_{0}\right) \gamma^{\mu} i S(p-w)\left(-i e_{0}\right) \gamma^{\sigma} i D_{\lambda \sigma}(w), \\
\left(-i e_{0}\right) \Lambda_{(1 S)}^{\mu}(k, p)= & \int_{\Lambda} \frac{d^{4} w}{(2 \pi)^{4}}\left(-i g_{Y}\right) \mathbf{1} i S(k-w) \\
& \times\left(-i e_{0}\right) \gamma^{\mu} i S(p-w)\left(-i g_{Y}\right) \mathbf{1} i \Delta_{S}(w), \\
\left(-i e_{0}\right) \Lambda_{(1 P)}^{\mu}(k, p)= & \int_{\Lambda} \frac{d^{4} w}{(2 \pi)^{4}}\left(-i g_{Y}\right) i \gamma_{5} i S(k-w) \\
& \times\left(-i e_{0}\right) \gamma^{\mu} i S(p-w)\left(-i g_{Y}\right) i \gamma_{5} i \Delta_{P}(w),
\end{aligned}
$$

and the self-energies

$$
\begin{aligned}
& i \Sigma_{(1 V)}(p)=\int_{\Lambda} \frac{d^{4} k}{(2 \pi)^{4}}\left(-i e_{0}\right) \gamma^{\mu} i S(k)\left(-i e_{0}\right) \gamma^{\nu} i D_{\mu \nu}(k-p), \\
& i \Sigma_{(1 S)}(p)=\int_{\Lambda} \frac{d^{4} k}{(2 \pi)^{4}}\left(-i g_{Y}\right) \mathbf{1} i S(k)\left(-i g_{Y}\right) \mathbf{1} i \Delta_{S}(k-p), \\
& i \Sigma_{(1 P)}(p)=\int_{\Lambda} \frac{d^{4} k}{(2 \pi)^{4}}\left(-i g_{Y}\right) i \gamma_{5} i S(k)\left(-i g_{Y}\right) i \gamma_{5} i \Delta_{P}(k-p) .
\end{aligned}
$$

Taking free massless fermion, scalar, and pseudoscalar propagators, and the photon propagator in the Feynman gauge $(a=1)$,

$$
S(p)=\frac{\hat{p}}{p^{2}}, \quad D_{\mu \nu}(q)=-\frac{g_{\mu \nu}}{q^{2}}, \quad \Delta_{S}(q)=\Delta_{P}(q)=\frac{1}{q^{2}}
$$

the one-loop vertices can be expressed as

$$
\begin{aligned}
\Lambda_{(1 V)}^{\mu}(k, p) & =2 e_{0}^{2} R^{\mu}(k, p)+2 e_{0}^{2} S^{\mu}(k, p), \\
\Lambda_{(1 S)}^{\mu}(k, p) & =-g_{Y}^{2} R^{\mu}(k, p)+g_{Y}^{2} S^{\mu}(k, p), \\
\Lambda_{(1 P)}^{\mu}(k, p) & =\Lambda_{(1 S)}^{\mu}(k, p),
\end{aligned}
$$

where the last identity is obtained form Eq. (A8) by using $\gamma_{5} \gamma^{\mu}=-\gamma^{\mu} \gamma_{5}$, and where

$$
\begin{aligned}
R^{\mu}(k, p) \equiv-i \int_{\Lambda} & \frac{d^{4} w}{(2 \pi)^{4}} \frac{\gamma^{\mu}(\hat{p}-\hat{w})(\hat{k}-\hat{w}) / 2-(\hat{k}-\hat{w})(\hat{p}-\hat{w}) \gamma^{\mu} / 2}{(k-w)^{2}(p-w)^{2} w^{2}} \\
S^{\mu}(k, p) \equiv-i \int_{\Lambda} & \frac{d^{4} w}{(2 \pi)^{4}}\left[\frac{(k-w) \cdot(p-w) \gamma^{\mu}}{(k-w)^{2}(p-w)^{2} w^{2}}-\frac{(k-w)^{\mu}(\hat{p}-\hat{w})}{(k-w)^{2}(p-w)^{2} w^{2}}\right. \\
& \left.-\frac{(p-w)^{\mu}(\hat{k}-\hat{w})}{(k-w)^{2}(p-w)^{2} w^{2}}\right]
\end{aligned}
$$

Thus the sum of one-loop vertex corrections, Eq. (A4), can be rewritten as

$$
\Lambda_{(1)}^{\mu}(k, p)=2\left[e_{0}^{2}-g_{Y}^{2}\right] R^{\mu}(k, p)+2\left[e_{0}^{2}+g_{Y}^{2}\right] S^{\mu}(k, p) .
$$

The sum of self-energy contributions, Eq. (A5), can be computed straightforwardly 


$$
\mathcal{Z}_{(1)}\left(p^{2}\right)=-\frac{\left(e_{0}^{2}+g_{Y}^{2}\right)}{16 \pi^{2}}\left[\ln \left(\frac{\Lambda^{2}}{-p^{2}}\right)+\frac{3}{2}\right],
$$

in Minkowskian formulation.

The vacuum polarization up to two-loop corrections can be expressed as

$$
\Pi\left(q^{2}\right)=\Pi_{(1)}\left(q^{2}\right)+\Pi_{(2 a)}\left(q^{2}\right)+\Pi_{(2 b)}\left(q^{2}\right),
$$

where

$$
\begin{aligned}
\Pi_{(1)}\left(q^{2}\right) & =-\frac{i e_{0}^{2}}{3 q^{2}} \int_{\Lambda} \frac{d^{4} k}{(2 \pi)^{4}} \frac{\operatorname{Tr}\left[P_{\mu \nu}(q) \gamma^{\nu}(\hat{k}+\hat{q}) \gamma^{\mu} \hat{k}\right]}{(k+q)^{2} k^{2}} \\
\Pi_{(2 a)}\left(q^{2}\right) & =-\frac{i e_{0}^{2}}{3 q^{2}} \int_{\Lambda} \frac{d^{4} k}{(2 \pi)^{4}} \frac{\operatorname{Tr}\left[\gamma_{\mu}(\hat{k}+\hat{q}) \gamma^{\mu} \hat{k}\right]}{(k+q)^{2} k^{2}}\left[\mathcal{Z}_{(1)}\left((k+q)^{2}\right)+\mathcal{Z}_{(1)}\left(k^{2}\right)\right], \\
\Pi_{(2 b)}\left(q^{2}\right) & =-\frac{i e_{0}^{2}}{3 q^{2}} \int_{\Lambda} \frac{d^{4} k}{(2 \pi)^{4}} \frac{\operatorname{Tr}\left[\gamma_{\mu}(\hat{k}+\hat{q}) \Lambda_{(1)}^{\mu}(k+q, k) \hat{k}\right]}{(k+q)^{2} k^{2}} .
\end{aligned}
$$

The one-loop vacuum polarization $\Pi_{(1)}$ can be computed straightforwardly by making use of the projector $P_{\mu \nu}(q)=g_{\mu \nu}-4 q_{\mu} q_{\nu} / q^{2}$, which by contraction with the vacuum polarization tensor eliminates the term in $\Pi_{\mu \nu}$ proportional to the $g_{\mu \nu}$ tensor. With this projector the quadratically divergent contribution to $\Pi_{\mu \nu}$, which is an artifact of a hard-cutoff regularization, is eliminated explicitly.[3] The result is the well-known one-loop vacuum polarization:

$$
\Pi_{(1)}\left(q^{2}\right)=\frac{\alpha_{0}}{3 \pi}\left[\ln \left(\frac{\Lambda^{2}}{q^{2}}\right)+\mathcal{O}(1)\right],
$$

with $q^{2}$ the Euclidean momentum and $\alpha_{0}=e_{0}^{2} / 4 \pi$.

The sum of the one-loop vertex functions is given in terms of the functions $R^{\mu}$ and $S^{\mu}$. The one-loop vertex in the Feynman gauge has been computed in Ref. 62, see also Ref. 63. for arbitrary gauge. One can show that, with $q^{2}=(k-p)^{2}$,

$$
\begin{aligned}
& 2 e_{0}^{2} R^{\mu}(k, p)=\Lambda_{(1 R)}^{\mu}(k, p), \\
& 2 e_{0}^{2} S^{\mu}(k, p)=\Lambda_{(1 L)}^{\mu}(k, p)+\Lambda_{(1 I)}^{\mu}(k, p),
\end{aligned}
$$

with

$$
\begin{aligned}
\Lambda_{(1 L)}^{\mu}(k, p) & \equiv \frac{\gamma^{\mu}}{2}\left[-\mathcal{Z}_{(1)}\left(k^{2}\right)-\mathcal{Z}_{(1)}\left(p^{2}\right)\right]+\frac{(k+p)^{\mu}(\hat{k}+\hat{p})}{2\left(k^{2}-p^{2}\right)}\left[-\mathcal{Z}_{(1)}\left(k^{2}\right)+\mathcal{Z}_{(1)}\left(p^{2}\right)\right], \\
\Lambda_{(1 I)}^{\mu}(k, p) & \equiv \tau_{2}\left(k^{2}, p^{2}, q^{2}\right) T_{2}^{\mu}(k, p)+\tau_{3}\left(k^{2}, p^{2}, q^{2}\right) T_{3}^{\mu}(k, p)+\tau_{6}\left(k^{2}, p^{2}, q^{2}\right) T_{6}^{\mu}(k, p), \\
\Lambda_{(1 R)}^{\mu}(k, p) & \equiv \tau_{8}\left(k^{2}, p^{2}, q^{2}\right) T_{8}^{\mu}(k, p),
\end{aligned}
$$

\footnotetext{
${ }^{13}$ The quadratically divergent contribution $\Lambda^{2} / q^{2}$ is a notorious artifact of computing vacuum polarization corrections in the presence of a hard cutoff (i.e., an explicit cutoff in the momentum integrations instead of Pauli-Villars regularization, see for a recent discussion Ref. [64]).
} 
where $\Lambda_{(1 L)}^{\mu}$ is the one-loop longitudinal part of the vertex, and where the $\tau_{i} T_{i}^{\mu}$, s are the one-loop transverse parts as defined and computed in Refs. [62, 63]. By construction, this Ball-Chiu expression for the longitudinal vertex $\Lambda_{(1 L)}^{\mu}$ satisfies the WTI 62]:

$$
q_{\mu} \Lambda_{(1 L)}^{\mu}(k, p)=-\hat{k} \mathcal{Z}_{(1)}\left(k^{2}\right)+\hat{p} \mathcal{Z}_{(1)}\left(p^{2}\right) .
$$

Using Eqs. (A18), (A23), (A25), and (A26), we write

$$
\Pi_{(2 b)}\left(q^{2}\right)=\left(1+\frac{g_{Y}^{2}}{e_{0}^{2}}\right)\left[\Pi_{(2 L)}\left(q^{2}\right)+\Pi_{(2 I)}\left(q^{2}\right)\right]+\left(1-\frac{g_{Y}^{2}}{e_{0}^{2}}\right) \Pi_{(2 R)}\left(q^{2}\right),
$$

where

$$
\Pi_{(2 j)}\left(q^{2}\right) \equiv-\frac{i e_{0}^{2}}{3 q^{2}} \int_{\Lambda} \frac{d^{4} k}{(2 \pi)^{4}} \frac{\operatorname{Tr}\left[\gamma_{\mu}(\hat{k}+\hat{q}) \Lambda_{(1 j)}^{\mu}(k+q, k) \hat{k}\right]}{(k+q)^{2} k^{2}}, \quad j=L, I, R .
$$

Since the one-loop transverse vertex functions themselves are finite, i.e., these function are independent of the cutoff $\Lambda$, the leading logarithmic contributions to the vacuum polarization result from integrations over momenta $k^{2} \gg q^{2}$ in $\Pi_{(2 R)}$ and $\Pi_{(2 I)}$. These leading logarithmic contribution can be found by first deriving the $k^{2} \gg q^{2}$ asymptotic behavior of the transverse structure functions given in [63], after which the integration over angles can be performed. In the Feynman gauge $a=1$, the asymptotic behavior $k^{2} \gg q^{2}$ of the $\tau$ 's is

$$
\begin{aligned}
& \tau_{2} \approx \frac{\alpha_{0}}{24 \pi} \frac{1}{k^{4}}, \quad \tau_{3} \approx \frac{\alpha_{0}}{6 \pi} \frac{1}{k^{2}} \ln \left(\frac{q^{2}}{k^{2}}\right)-\frac{29}{72} \frac{\alpha_{0}}{\pi} \frac{1}{k^{2}}, \\
& \tau_{6} \approx \frac{\left(2 k \cdot q+q^{2}\right)}{2} \frac{\alpha_{0}}{24 \pi} \frac{1}{k^{4}}, \quad \tau_{8} \approx-\frac{\alpha_{0}}{2 \pi} \frac{1}{k^{2}},
\end{aligned}
$$

where $k^{2}$ and $q^{2}$ are Minskowskian momenta. By making use of these asymptotic expressions the integration over angles in $\Pi_{(2 R)}$ and $\Pi_{(2 I)}$ can be performed straightforwardly, after performing a Wick rotation. The integrations over momenta $k^{2} \geq q^{2}$ leads to logarithmic corrections. The result reads (in Euclidean formulation):

$$
\begin{aligned}
& \Pi_{(2 I)}\left(q^{2}\right)=\frac{\alpha_{0}^{2}}{\pi^{2}}\left[\frac{1}{24} \ln ^{2}\left(\frac{\Lambda^{2}}{q^{2}}\right)+\frac{29}{144} \ln \left(\frac{\Lambda^{2}}{q^{2}}\right)+\mathcal{O}(1)\right], \\
& \Pi_{(2 R)}\left(q^{2}\right)=\frac{\alpha_{0}^{2}}{\pi^{2}}\left[\frac{1}{4} \ln \left(\frac{\Lambda^{2}}{q^{2}}\right)+\mathcal{O}(1)\right] .
\end{aligned}
$$

The logarithmic corrections of $\tau_{2}$ and $\tau_{6}$ cancel each other, and the contributions of $\tau_{3}$ give rise to a $\ln ^{2}$ term.

An analogous computation can be performed for the self-energy and longitudinal vertex corrections. Due to the Ball-Chiu expression (A27) for $\Lambda_{(1 L)}^{\mu}$, the contributions $\Pi_{(2 a)}$ and $\Pi_{(2 L)}$ depend on the one-loop computation of the self-energy $\mathcal{Z}_{(1)}$ given in Eq. (A19). After expanding $\mathcal{Z}_{(1)}\left((k+q)^{2}\right)$ for $k^{2} \gg q^{2}$,

$$
\begin{aligned}
\mathcal{Z}_{(1)}\left((k+q)^{2}\right) & \approx \mathcal{Z}_{(1)}\left(k^{2}\right)+\left(2 k \cdot q+q^{2}\right) \mathcal{Z}_{(1)}^{\prime}\left(k^{2}\right) \\
& +\frac{1}{2}\left(2 k \cdot q+q^{2}\right)^{2} \mathcal{Z}_{(1)}^{\prime \prime}\left(k^{2}\right)+\frac{1}{6}\left(2 k \cdot q+q^{2}\right)^{3} \mathcal{Z}_{(1)}^{\prime \prime \prime}\left(k^{2}\right)
\end{aligned}
$$


and using that $\mathcal{Z}_{(1)}\left((k+q)^{2}\right) \approx \mathcal{Z}_{(1)}\left(q^{2}\right)$ for $q^{2} \gg k^{2}$, the angular integration can be performed, and the logarithmic corrections can be computed. The result is

$$
\begin{aligned}
\Pi_{(2 a)}\left(q^{2}\right)+\left(1+\frac{\lambda_{Y}}{\alpha_{0}}\right) \Pi_{(2 L)}\left(q^{2}\right) & =\frac{\alpha_{0}\left(\alpha_{0}+\lambda_{Y}\right)}{\pi^{2}} \\
& \times\left[-\frac{1}{24} \ln ^{2}\left(\frac{\Lambda^{2}}{q^{2}}\right)-\frac{29}{144} \ln \left(\frac{\Lambda^{2}}{q^{2}}\right)+\mathcal{O}(1)\right]
\end{aligned}
$$

with $\alpha_{0}=e_{0}^{2} / 4 \pi$ and $\lambda_{Y}=g_{Y}^{2} / 4 \pi$. Thus, comparing this expression with the $\Pi_{(2 I)}$ term in Eq. (A31), we see that the "overlapping divergencies" (i.e., the $\ln ^{2}$ ) cancel

$$
\Pi_{(2 a)}\left(q^{2}\right)+\left(1+\frac{\lambda_{Y}}{\alpha_{0}}\right)\left[\Pi_{(2 L)}\left(q^{2}\right)+\Pi_{(2 I)}\left(q^{2}\right)\right]=\frac{\alpha_{0}\left(\alpha_{0}+\lambda_{Y}\right)}{\pi^{2}} \mathcal{O}(1) .
$$

Such a cancellation occurs in a similar manner in any covariant gauge $a$. Thus, the twoloop contribution contribution to $\Pi$ is described solely by the part of the transverse vertex containing the $T_{8}^{\mu}$ tensor ${ }^{\mathbb{4}}$ i.e., $\Pi_{(2 R)}$, and, after adding all the pieces, we deduce that

$$
\Pi\left(q^{2}\right)=\frac{\alpha_{0}}{2 \pi}\left(\frac{2}{3}+\frac{\alpha_{0}}{2 \pi}-\frac{\lambda_{Y}}{2 \pi}\right) \ln \left(\frac{\Lambda^{2}}{q^{2}}\right)+\left(\alpha_{0} / \pi\right) \mathcal{O}(1) .
$$

\section{APPENDIX B: THE JOHNSON-WILLEY-BAKER EQUATION}

In this appendix we derive the equation

$$
f_{(1)}=\frac{N \alpha_{0}}{\pi}\left[\frac{2}{3}+\frac{\phi_{1}+\phi_{2}\left(2+\phi_{2}\right)}{1-\phi_{1}}+\phi_{3}\right]
$$

for the $f_{(1)}$ function given by Eq. (64), with the functions $\phi_{j}$ given by Eqs. (71) $-(73)$. The derivation of Eq. (B1) was given (for pure QED) by Johnson, Willey, and Baker (JWB) in Ref. [56]. Since their result is formulated in terms of the BS fermion-fermion scattering kernel $K^{(2)}$ their method is also applicable to the GNJL model.

In order to derive the result of [56], the following is assumed.

- The fermion wave function equals one, $\mathcal{Z}=1 / A=1$, in the Landau gauge. In principle, this assumption is redundant since the JWB result is valid in any gauge.

- Internal photon propagators are replaced by their canonical form $\Delta(q)=1 / q^{2}$ which is self-consistent in the neighborhood of an UV stable fixed point. Only a single fermion loop, thus a single power of $\ln \Lambda$ contributes to the vacuum polarization.

\footnotetext{
${ }^{14}$ As was shown in 63, this particular transverse structure function $\tau_{8}$ does not depend on the gauge parameter $a$.
} 
- Translational invariance of naively logarithmically divergent and finite momentum space integrals is assumed. Implicitly, use is made of invariance under chargeconjugation $(C)$ and parity-time $(P T)$ transformations.

- Also it is assumed that we are in the scaling region of the theory, where the only relevant dimensionless variable is $q^{2} / \Lambda^{2}$. We consider short distances with respect to the IR length scale $\xi \sim 1 /\left|m_{\sigma}\right|$, thus $\left|m_{\sigma}\right|^{2} \leq q^{2} \ll \Lambda^{2}$.

Hence,

$$
\mathcal{Z}\left(k^{2}\right)=1 / A\left(k^{2}\right)=1, \quad S(k)=\frac{\hat{k}}{k^{2}}, \quad \Gamma^{\mu}(k, k)=\gamma^{\mu}
$$

The vacuum polarization tensor is

$$
\Pi^{\mu \nu}(q)=\frac{i \alpha_{0}}{4 \pi^{3}} \int_{\Lambda} d^{4} k \tilde{\operatorname{T}} r\left[S(k+q) \Gamma^{\mu}(k+q, k) S(k) \gamma^{\nu}\right]
$$

where $\tilde{T}$ r denotes the sum over both spinor and flavor indices. Since, in the chiral symmetric phase, the $N$ fermions are degenerate, all $N$ fermion propagators and $N$ photon-fermion vertices are degenerate. Hence, the sum over flavor indices gives rise to a factor $N$, i.e., $\tilde{\mathrm{T}} \mathrm{r} \rightarrow N \mathrm{Tr}$, where $\mathrm{Tr}$ is the sum over spinor indices.

Since vacuum polarization tensor is transverse and the only relevant momentum variable is $q^{2} / \Lambda^{2} \ll 1$, the equation for the vacuum polarization can be written as

$$
\Pi\left(q^{2}\right)=-\frac{1}{6} \frac{q^{\mu} q^{\nu}}{q^{2}} \frac{\partial^{2}}{\partial q_{\alpha} q^{\alpha}} \Pi_{\mu \nu}(q)+\mathcal{O}(1)+\mathcal{O}\left((q / \Lambda)^{\sigma}\right),
$$

where $\sigma$ is some positive power. After inserting Eq. (B33), and setting $q^{2}=0$ in the integrand, and using $q$ as the infrared cutoff in the momentum integral, we obtain

$$
\begin{aligned}
\Pi\left(q^{2}\right) \approx-\frac{1}{6} & \frac{q_{\mu} q_{\nu}}{q^{2}} \frac{i N \alpha_{0}}{4 \pi^{3}} \int_{q, \Lambda} d^{4} k \operatorname{Tr}\left[S_{\alpha}^{\alpha}(k) \Gamma^{\mu}(k) S(k) \gamma^{\nu}\right. \\
& \left.+2 S_{\alpha}(k) \Gamma^{\mu, \alpha}(k) S(k) \gamma^{\nu}+S(k) \Gamma_{\alpha}^{\mu, \alpha}(k) S(k) \gamma^{\nu}\right],
\end{aligned}
$$

where the derivatives are defined as follows (with $\left.\Gamma^{\mu}(k) \equiv \Gamma^{\mu}(k, k)\right)$ :

$$
\begin{gathered}
S_{\alpha}(k) \equiv \frac{\partial}{\partial k^{\alpha}} S(k)=-\frac{\hat{k} \gamma_{\alpha} \hat{k}}{k^{4}}, \quad S_{\alpha}^{\alpha}(k) \equiv \frac{\partial^{2}}{\partial k_{\alpha} \partial k^{\alpha}} S(k)=-\frac{4 \hat{k}}{k^{4}} \\
\left.\Gamma_{\mu, \alpha}(k) \equiv \frac{\partial}{\partial q^{\alpha}} \Gamma_{\mu}(k+q, k)\right|_{q=0},\left.\quad \Gamma_{\mu, \alpha}^{\alpha}(k) \equiv \frac{\partial^{2}}{\partial q_{\alpha} \partial q^{\alpha}} \Gamma_{\mu}(k+q, k)\right|_{q=0} .
\end{gathered}
$$

Since the integral Eq. (B5) can only be proportional to $g^{\mu \nu}$, it reduces to

$$
\begin{aligned}
\Pi\left(q^{2}\right) \approx-\frac{i N \alpha_{0}}{96 \pi^{3}} \int_{q, \Lambda} d^{4} k \operatorname{Tr}\left[S_{\alpha}^{\alpha}(k) \Gamma^{\mu}(k) S(k) \gamma_{\mu}\right. \\
\left.+2 S_{\alpha}(k) \Gamma^{\mu, \alpha}(k) S(k) \gamma_{\mu}+S(k) \Gamma_{\alpha}^{\mu, \alpha}(k) S(k) \gamma_{\mu}\right]
\end{aligned}
$$

The SDE for the vertex $\Gamma^{\mu}$ reads, in terms of $K^{(2)}$ (see Fig. 6) 


$$
\begin{aligned}
\delta_{i j} \Gamma_{a b}^{\mu}(k+q, k) & =\delta_{i j} \gamma_{a b}^{\mu}+i e_{0}^{2} \int_{\Lambda} \frac{d^{4} p}{(2 \pi)^{4}}\left[S(p+q) \delta_{n m} \Gamma^{\mu}(p+q, p) S(p)\right]_{d c} \\
& \times K_{\substack{c d, a b \\
m n, i j}}^{(2)}(p, p+q, k+q) .
\end{aligned}
$$

From now on we omit spinor and flavor indices. Differentiating now the SDE Eq. (BB9) with respect to $q$, and setting $q=0$ for the integrand and $q$ as IR cutoff, we obtain

$$
\begin{aligned}
\Gamma_{\mu, \alpha}(k) & =i e_{0}^{2} \int_{q, \Lambda} \frac{d^{4} p}{(2 \pi)^{4}}\left[S(p) \Gamma_{\mu, \alpha}(p) S(p) K^{(2)}(p, k)\right. \\
& \left.+S_{\alpha}(p) \Gamma_{\mu}(p) S(p) K^{(2)}(p, k)+S(p) \Gamma_{\mu}(p) S(p) K_{\alpha}^{(2)}(p, k)\right]
\end{aligned}
$$

and for the second derivative of the vertex, we find

$$
\begin{aligned}
\Gamma_{\alpha}^{\mu, \alpha}(k)= & i e_{0}^{2} \int_{q, \Lambda} \frac{d^{4} p}{(2 \pi)^{4}}\left[S(p) \Gamma_{\alpha}^{\mu, \alpha}(p) S(p) K^{(2)}(p, k)+2 S_{\alpha}(p) \Gamma^{\mu, \alpha}(p) S(p) K^{(2)}(p, k)\right. \\
& +S_{\alpha}^{\alpha}(p) \Gamma^{\mu}(p) S(p) K^{(2)}(p, k)+2 S(p) \Gamma^{\mu, \alpha}(p) S(p) K_{\alpha}^{(2)}(p, k) \\
& \left.+2 S^{\alpha}(p) \Gamma^{\mu}(p) S(p) K_{\alpha}^{(2)}(p, k)+S(p) \Gamma^{\mu}(p) S(p) K_{\alpha}^{(2) \alpha}(p, k)\right]
\end{aligned}
$$

where $K^{(2)}(p, k)$ and the derivatives $K_{\alpha}^{(2)}(p, k)$ and $K_{\alpha}^{(2) \alpha}(p, k)$ are defined in Eqs. (74)-(76).

The first derivative of the vertex is antisymmetric in $\alpha$ and $\mu$, because of the assumption Eq. (B) . Furthermore, $C$ and $P T$ invariance imply that the only nonzero contribution to the first derivative of $\Gamma^{\mu}$ (with $q=0$ and $\Lambda \rightarrow \infty$ ) must be proportional to the tensor $\left(\gamma_{\mu} \hat{k} \gamma_{\alpha}-\gamma_{\alpha} \hat{k} \gamma_{\mu}\right)$. Thus we write

$$
\begin{aligned}
\Gamma_{[\mu, \alpha]}(k) & \equiv \Gamma_{\mu, \alpha}(k)-\Gamma_{\alpha, \mu}(k)=\frac{\left(\gamma_{\mu} \hat{k} \gamma_{\alpha}-\gamma_{\alpha} \hat{k} \gamma_{\mu}\right)}{k^{2}} \Gamma^{\prime}, \\
\Gamma_{(\mu, \alpha)}(k) & \equiv \Gamma_{\mu, \alpha}(k)+\Gamma_{\alpha, \mu}(k)=S_{\mu \alpha}^{-1}(k),
\end{aligned}
$$

where $\Gamma^{\prime}$ is a dimensionless scalar function. ${ }^{\prime \prime}$ Since $S_{\nu \mu}^{-1}(k)=0$, due to the WTI for the vertex and Eq. (B2), we find that

$$
\Gamma_{\mu, \alpha}(k)=\frac{1}{2} \Gamma_{[\mu, \alpha]}(k)=\frac{\left(\gamma_{\mu} \hat{k} \gamma_{\alpha}-\gamma_{\alpha} \hat{k} \gamma_{\mu}\right)}{2 k^{2}} \Gamma^{\prime} .
$$

After some algebra (taking traces over spinor and flavor indices), we can derive from Eqs. (B10) and (B14) that

$$
\begin{aligned}
\lim _{q \rightarrow 0, \Lambda \rightarrow \infty} \Gamma_{\mu, \alpha}(k) & =\left(\phi_{1}+\phi_{2}+\phi_{1} \lim _{q \rightarrow 0, \Lambda \rightarrow \infty} \Gamma^{\prime}\right) \frac{\left(\gamma_{\mu} \hat{k} \gamma_{\alpha}-\gamma_{\alpha} \hat{k} \gamma_{\mu}\right)}{2 k^{2}} \Longrightarrow \\
\lim _{q \rightarrow 0, \Lambda \rightarrow \infty} \Gamma^{\prime} & =\frac{\phi_{1}+\phi_{2}}{1-\phi_{1}},
\end{aligned}
$$

\footnotetext{
15 The function $\Gamma^{\prime}$ is related to the transverse structure function $\tau_{8}\left(k^{2}, k^{2}, 0\right)$ of Ref. [63]. At the one-loop level $\Gamma_{(1)}^{\prime}=-k^{2} \tau_{8}\left(k^{2}, k^{2}, 0\right)$.
} 
where the $\phi_{j}$ functions are defined in Eqs. (71)-(73).

Since $\Gamma^{\mu}(k)=\gamma^{\mu}$, and using Eq. (B11) for $\Gamma_{\alpha}^{\mu, \alpha}(k)$, the second derivative of $\Gamma_{\alpha}^{\mu, \alpha}$ in Eq. (B8) can be eliminated. The result is

$$
\Pi\left(q^{2}\right)=\frac{N \alpha_{0}}{2 \pi}\left[\sum_{n=1}^{5} I_{n}\left(q^{2} / \Lambda^{2}\right)+\mathcal{O}\left(q^{2} / \Lambda^{2}\right)\right]
$$

where

$$
\begin{aligned}
& I_{1} \equiv-\frac{i}{48} \int_{q, \Lambda} \frac{d^{4} k}{\pi^{2}} \tilde{\operatorname{Tr}}\left[S_{\alpha}^{\alpha}(k) \gamma^{\mu} S(k) \gamma_{\mu}\right] \\
& I_{2} \equiv-\frac{i}{24} \int_{q, \Lambda} \frac{d^{4} k}{\pi^{2}} \tilde{\operatorname{Tr}}\left[S_{\alpha}(k) \Gamma^{\mu, \alpha}(k) S(k) \gamma_{\mu}\right], \\
& I_{3} \equiv \frac{e_{0}^{2}}{24} \int_{q, \Lambda} \frac{d^{4} k}{\pi^{2}} \int_{q, \Lambda} \frac{d^{4} p}{(2 \pi)^{4}} \tilde{\operatorname{Tr}}\left[S(p) \Gamma_{\mu, \alpha}(p) S(p) K^{(2) \alpha}(p, k) S(k) \gamma^{\mu} S(k)\right], \\
& I_{4} \equiv \frac{e_{0}^{2}}{24} \int_{q, \Lambda} \frac{d^{4} k}{\pi^{2}} \int_{q, \Lambda} \frac{d^{4} p}{(2 \pi)^{4}} \tilde{\operatorname{Tr}}\left[S_{\alpha}(p) \gamma_{\mu} S(p) K^{(2) \alpha}(p, k) S(k) \gamma^{\mu} S(k)\right], \\
& I_{5} \equiv \frac{e_{0}^{2}}{48} \int_{q, \Lambda} \frac{d^{4} k}{\pi^{2}} \int_{q, \Lambda} \frac{d^{4} p}{(2 \pi)^{4}} \tilde{\operatorname{Tr}}\left[S(p) \gamma_{\mu} S(p) K_{\alpha}^{(2) \alpha}(p, k) S(k) \gamma^{\mu} S(k)\right] .
\end{aligned}
$$

Using translational invariance, $C$-PT invariance, Eqs. (71)-(73) and (B15), we can derive that

$$
\begin{aligned}
& I_{1}=\frac{2}{3} \int_{q^{2}}^{\Lambda^{2}} \frac{d k^{2}}{k^{2}}+\mathcal{O}(1), \quad I_{2}=\left[\frac{\phi_{1}+\phi_{2}}{1-\phi_{1}}\right] \int_{q^{2}}^{\Lambda^{2}} \frac{d k^{2}}{k^{2}}+\mathcal{O}(1), \\
& I_{3}=\phi_{2}\left[\frac{\phi_{1}+\phi_{2}}{1-\phi_{1}}\right] \int_{q^{2}}^{\Lambda^{2}} \frac{d k^{2}}{k^{2}}+\mathcal{O}(1), \quad I_{4}=\phi_{2} \int_{q^{2}}^{\Lambda^{2}} \frac{d k^{2}}{k^{2}}+\mathcal{O}(1), \quad I_{5}=\phi_{3} \int_{q^{2}}^{\Lambda^{2}} \frac{d k^{2}}{k^{2}}+\mathcal{O}(1),
\end{aligned}
$$

where $q^{2}$ is the Euclidean momentum. Substituting Eqs. (B22) in Eq. (B16), we get

$$
\Pi\left(q^{2}\right)=\frac{N \alpha_{0}}{2 \pi}\left[\frac{2}{3}+\frac{\phi_{1}+\phi_{2}\left(2+\phi_{2}\right)}{1-\phi_{1}}+\phi_{3}\right] \ln \frac{\Lambda^{2}}{q^{2}}+\left(N \alpha_{0} / \pi\right) \mathcal{O}(1) .
$$

With Eq. (64), we obtain Eq. (B1). This is the main result of Ref. [56. The entire derivation did not yet specify the BS kernel $K^{(2)}$. Therefore Eq. (B23) is applicable to the GNJL model as well. 


\section{REFERENCES}

[1] M. Gell-Mann and F.E. Low, Phys. Rev. 95, 1300 (1954).

[2] L.D. Landau and I.Ya. Pomeranchuk, Dokl. Akad. Nauk. 102, 489 (1955).

[3] E.S. Fradkin, Sov. Phys. JETP 28, 750 (1955).

[4] K.G. Wilson, Phys. Rev. B 4, 3174, 3184 (1971).

[5] K.G. Wilson and J.B. Kogut, Phys. Rep. C 12, 75 (1974).

[6] R. Fukuda and T. Kugo, Nucl. Phys. B117, 250 (1976).

[7] P.I. Fomin and V.A. Miransky, Phys. Lett. B 64, 166 (1976).

[8] P.I. Fomin, V.P. Gusynin, V.A. Miransky, and Yu.A. Sitenko, Nucl. Phys. B110, 445 (1976).

[9] P.I. Fomin, V.P. Gusynin, and V.A. Miransky, Phys. Lett. B 78, 136 (1978).

[10] P.I. Fomin, V.P. Gusynin, V.A. Miransky, and Yu.A. Sitenko, Riv. Nuovo Cimento 6, 1 (1983).

[11] V.A. Miransky, Nuovo Cimento A 90, 149 (1985).

[12] J.B. Kogut, E. Dagotto, and A. Kocić, Phys. Rev. Lett. 60, 772 (1988).

[13] J.B. Kogut, E. Dagotto, and A. Kocić, Nucl. Phys. B317, 271 (1989).

[14] J.B. Kogut, E. Dagotto, and A. Kocić, Nucl. Phys. B317, 253 (1989).

[15] W.A. Bardeen, C.N. Leung, and S.T. Love, Phys. Rev. Lett. 56, 1230 (1986).

[16] C.N. Leung, S.T. Love, and W.A. Bardeen, Nucl. Phys. B273, 649 (1986).

[17] K-I. Kondo, H. Mino, and K. Yamawaki, Phys. Rev. D 39, 2430 (1989).

[18] T. Appelquist, M. Soldate, T. Takeuchi, and L.C.R. Wijewardhana, In Proceedings of John Hopkins Workshop On Current Problems in Particle Theory, edited by G. Domokos and S. Kovesi-Domokos (World Scientific, Singapore, 1988).

[19] A. Kocić, S. Hands, J.B. Kogut, and E. Dagotto, Nucl. Phys. B347, 217 (1990).

[20] A. Kocić, J.B. Kogut, M-P. Lombardo, and K.C. Wang, Nucl. Phys. B397, 451 (1993).

[21] V. Azcoiti, G. Di Carlo, A. Galante, A.F. Grillo, V. Laliena, and C.E. Piedrafita, Phys. Lett. B 355, 270 (1995).

[22] W.A. Bardeen, S.T. Love, and V.A. Miransky, Phys. Rev. D 42, 3514 (1990).

[23] T. Appelquist, K. Lane, and U. Mahanta, Phys. Rev. Lett. 61, 1553 (1988).

[24] B. Holdom, Phys. Rev. Lett. 62, 997 (1989).

[25] K-I. Aoki, K. Morikawa, J-I. Sumi, H. Terao, and M. Tomoyose, Prog. Theor. Phys. 97, 479 (1997).

[26] K-I. Aoki, In Proceedings of International Workshop on Perspectives of Strong Coupling Gauge Theories, Nagoya, 1996, edited by K. Yamawaki (World Scientific, Singapore, 1996), p. 479, hep-ph/9706204.

[27] V.P. Gusynin, Mod. Phys. Lett. A 5, 133 (1990).

[28] E.V. Gorbar, Ukr. Fiz. Zh. 35, 982 (1990).

[29] P.E.L. Rakow, Nucl. Phys. B356, 27 (1991).

[30] K-I. Kondo, Nucl. Phys. B351, 259 (1991).

[31] K-I. Kondo, Int. J. Mod. Phys. A 6, 5447 (1991).

[32] V.P. Gusynin, Ukr. Phys. J. 36, 1819 (1991).

[33] E.V. Gorbar and E. Sausedo, Ukr. Phys. J. 36, 1025 (1991).

[34] M. Göckeler, R. Horsley, V. Linke, P.E.L. Rakow, G. Schierholz, and H. Stüben, Nucl. Phys. B487, 313 (1997).

[35] A. Kocić, J.B. Kogut, and K.C. Wang, Nucl. Phys. B398, 405 (1993). 
[36] S.J. Hands, A. Kocić, J.B. Kogut, R.L. Renken, D.K. Sinclair, and K.C. Wang, Nucl. Phys. B413, 503 (1994).

[37] E. Dagotto, A. Kocić, and J.B. Kogut, Phys. Lett. B 232, 235 (1989).

[38] V. Azcoiti, G. Di Carlo, and A.F. Grillo, Int. J. Mod. Phys. A 8, 4235 (1993).

[39] V. Azcoiti, G. Di Carlo, A. Galante, A.F. Grillo, V. Laliena, and C.E. Piedrafita, Phys. Lett. B 353, 279 (1995).

[40] M. Göckeler, R. Horsley, E. Laermann, P. Rakow, G. Schierholz, R. Sommer, and U.-J. Wiese, Phys. Lett. B 251, 567 (1990).

[41] M. Göckeler, R. Horsley, P.E.L. Rakow, G. Schierholz, and R. Sommer, Nucl. Phys. B371, 713 (1992).

[42] M. Göckeler, R. Horsley, V. Linke, P.E.L. Rakow, G. Schierholz, and H. Stüben, Phys. Rev. Lett. 80, 4119 (1998).

[43] V.A. Miransky, Dynamical Symmetry Breaking in Quantum Field Theories (WorldScientific, Singapore, 1993).

[44] M. Reenders, Ph.D. thesis, University of Groningen, 1999, hep-th/9906034.

[45] T. Appelquist, J. Terning, and L.C.R. Wijewardhana, Phys. Rev. D 44, 871 (1991).

[46] S. Hands, A. Kocić, and J.B. Kogut, Ann. Phys. (N.Y.) 224, 29 (1993).

[47] V.P. Gusynin and M. Reenders, Phys. Rev. D 57, 6356 (1998).

[48] K-I. Kondo, M. Tanabashi, and K. Yamawaki, Prog. Theor. Phys. 89, 1249 (1993).

[49] D.J. Amit, Field Theory, the Renormalization Group, and Critical Phenomena (World Scientific, Singapore, 1989).

[50] N. Goldenfeld, Lectures on Phase Transitions and the Renormalization Group (AddisonWesley, Reading, MA, 1992).

[51] J. Zinn-Justin, Quantum Field Theory and Critical Phenomena (Clarendon, Oxford, 1989).

[52] A. Kocić and J.B. Kogut, Nucl. Phys. B422, 593 (1994).

[53] J.D. Bjorken and S.D. Drell, Relativistic Quantum Fields (McGraw-Hill, New York, 1965).

[54] G. 't Hooft, Nucl. Phys. B75, 461 (1974).

[55] M. Harada, Y. Kikukawa, T. Kugo, and H. Nakano, Prog. Theor. Phys. 92, 1161 (1994).

[56] K. Johnson, R. Willey, and M. Baker, Phys. Rev. 163, 1699 (1967).

[57] M. Baker and K. Johnson, Phys. Rev. 183, 1292 (1969).

[58] K. Johnson and M. Baker, Phys. Rev. D 8, 1110 (1973).

[59] S.L. Adler, Phys. Rev. D 5, 3021 (1972).

[60] A.P. Prudnikov, Yu.A. Brychkov, and O.I. Marichev, Integrals and Series, Volume 2: Special Functions (Gordon and Breach, New York, 1986).

[61] V.A. Miransky and K. Yamawaki, Phys. Rev. D 55, 5051 (1997).

[62] J.S. Ball and T-W. Chiu, Phys. Rev. D 22, 2542 (1980).

[63] A. Kizilersü, M. Reenders, and M.R. Pennington, Phys. Rev. D 52, 1242 (1995).

[64] K. Fujikawa, Nucl. Phys. B428, 169 (1994). 


\section{FIGURES}

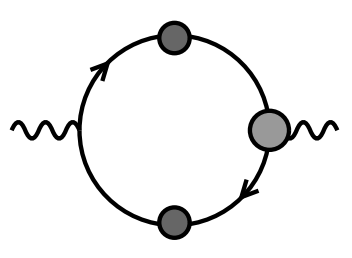

FIG. 1. The vacuum polarization tensor $\Pi_{\mu \nu}(q)=\left(-q^{2} g_{\mu \nu}+q_{\mu} q_{\nu}\right) \Pi\left(q^{2}\right)$, with the blobs representing full fermion propagators and a full photon-fermion vertex.

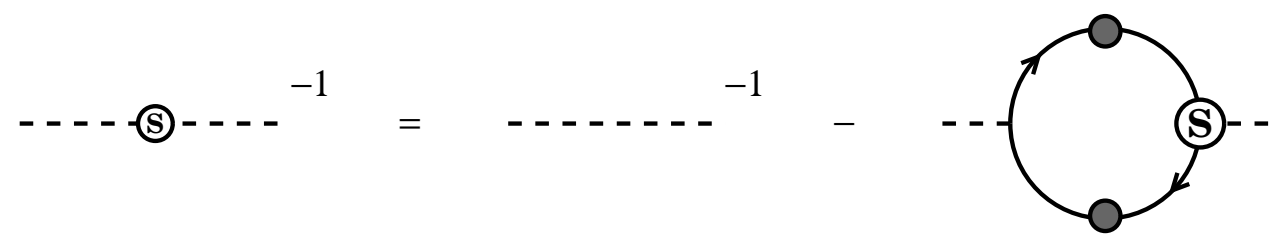

FIG. 2. SDE for the scalar propagator or $\sigma$ boson $\Delta_{S}(q)$.

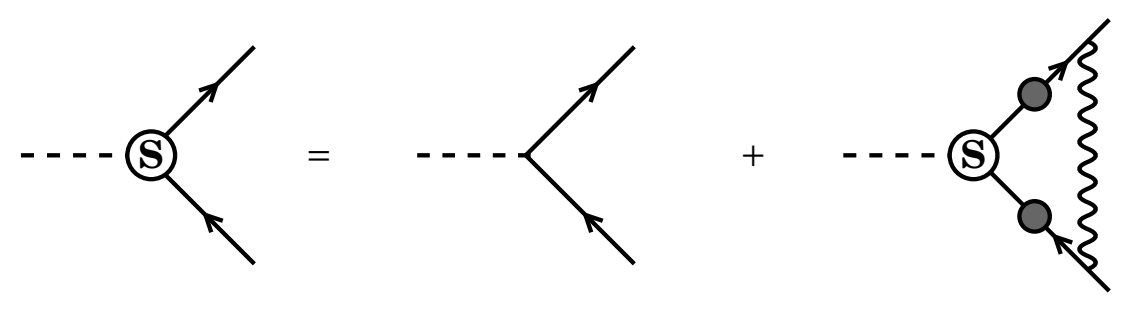

FIG. 3. SDE for the scalar vertex or Yukawa vertex $\Gamma_{S}(p+q, p)$ in the quenched-ladder approximation. 

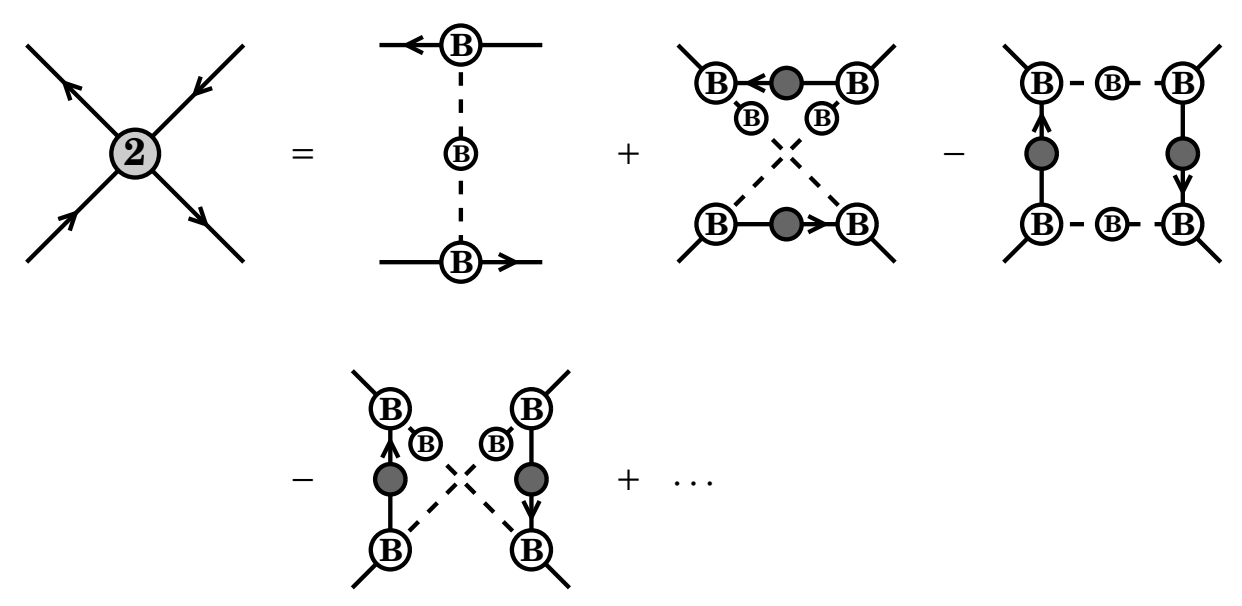

FIG. 4. Skeleton expansion for the BS kernel $K^{(2)}$.

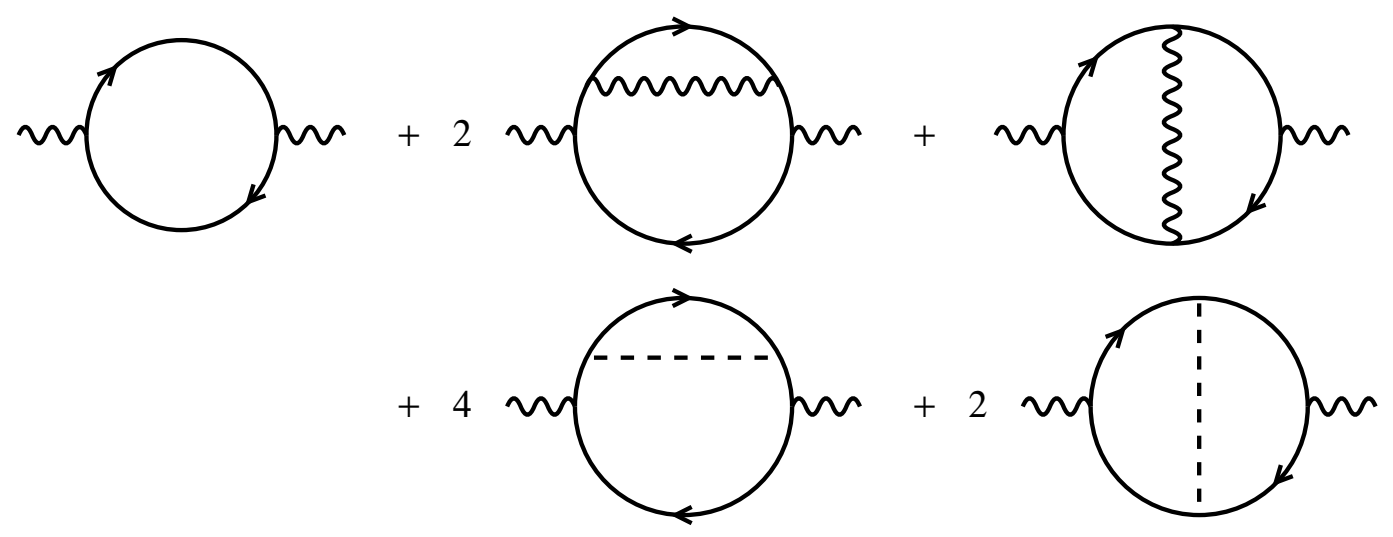

FIG. 5. One and two-loop vacuum polarization corrections containing both an internal photon (wavy) and a $\sigma$ and $\pi$ exchange (dashed). The contribution of $\pi$ equals the contribution of $\sigma$.

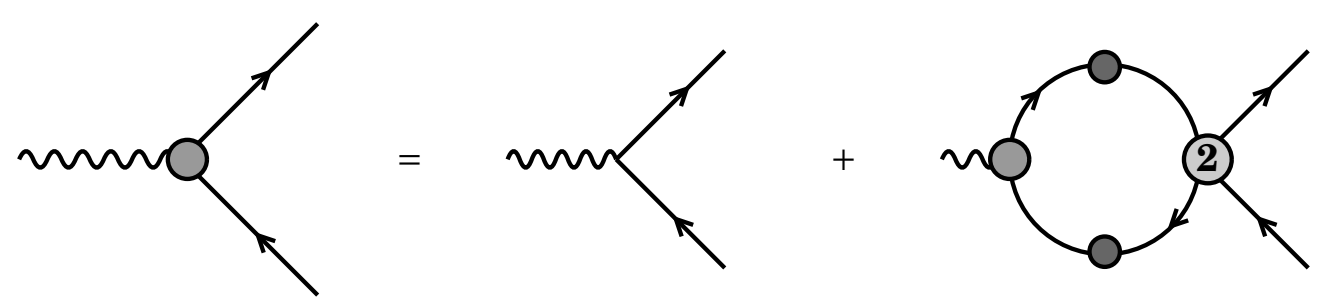

FIG. 6. SDE for the full photon-fermion vertex $\Gamma^{\mu}(k, p)$. 

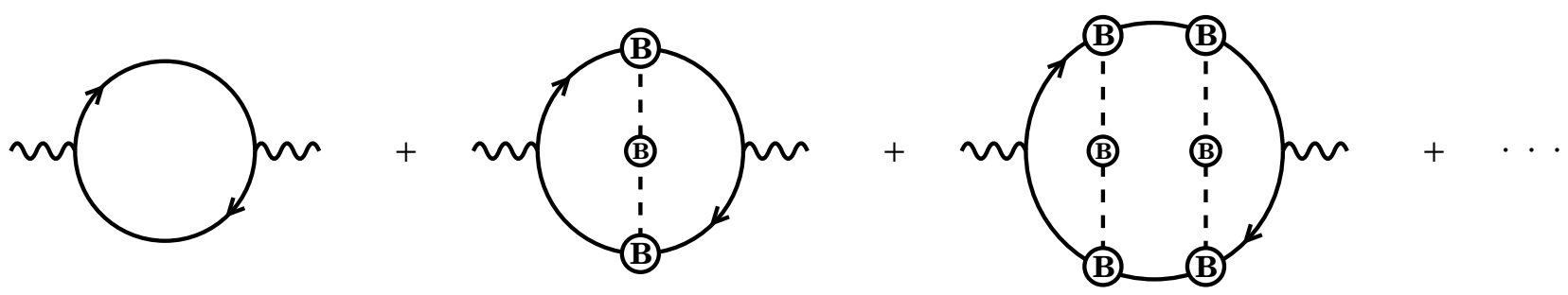

FIG. 7. Planar skeleton contributions to the vacuum polarization; the blobs represent both photon and $\sigma$ and $\pi$ exchanges.

The functions $\zeta_{1}$ and $\zeta_{3}$

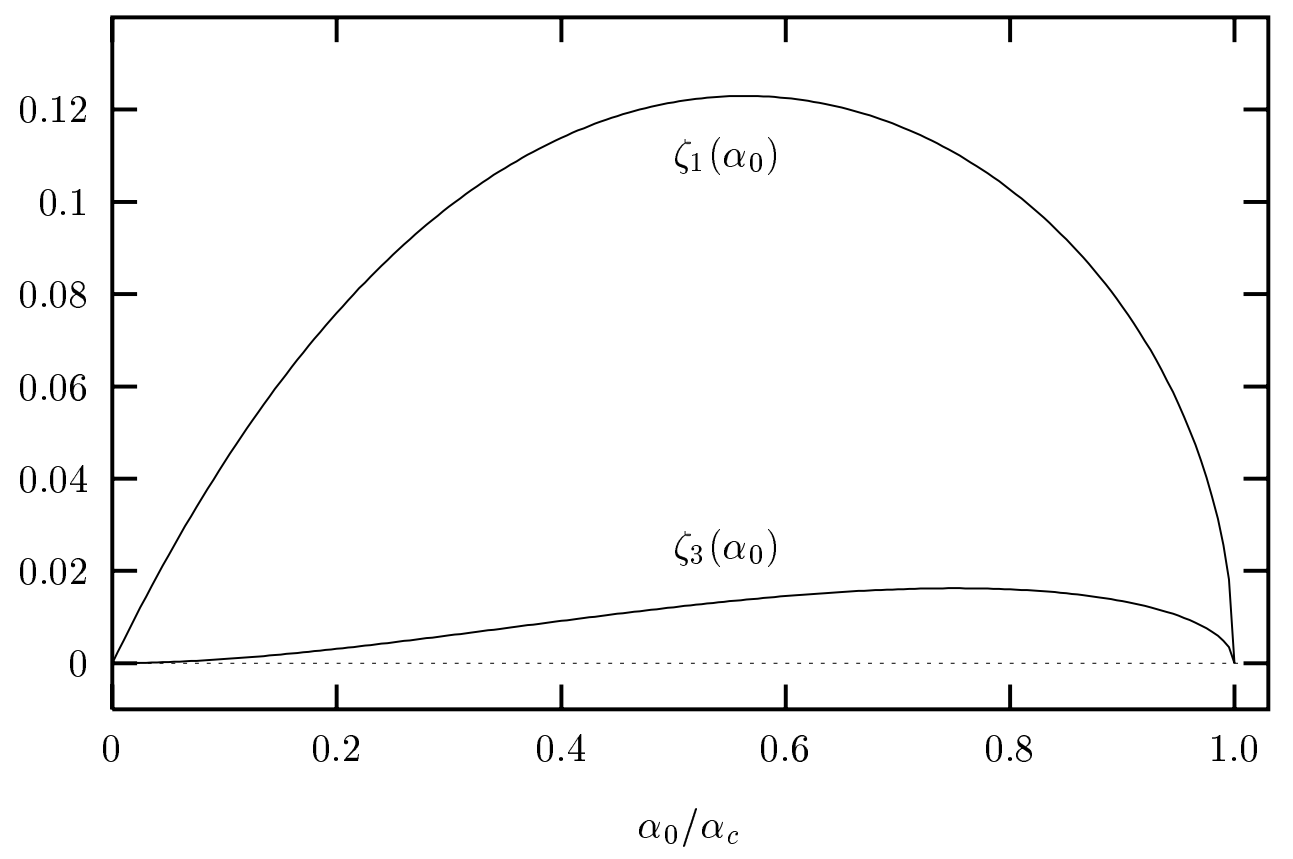

FIG. 8. The functions $\zeta_{1}$ and $\zeta_{3}$ plotted versus $\alpha_{0} / \alpha_{c}$. 
The $\beta$ function for $N=50,54,55,60$

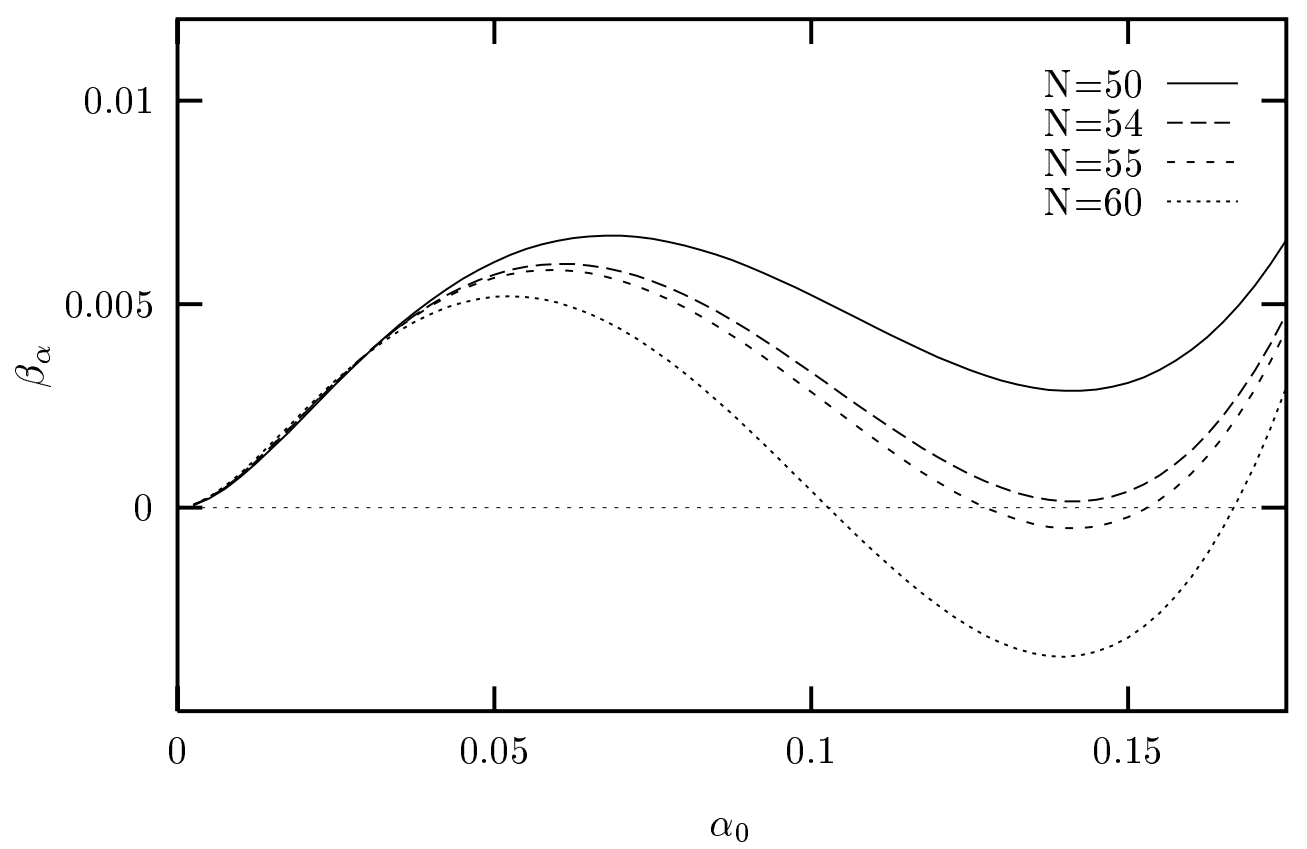

FIG. 9. Plot of the function $\beta_{\alpha}$ versus the gauge coupling $\alpha_{0}$ for various values of the fermion flavor number $N$.

The $\beta$ function for $N=60$

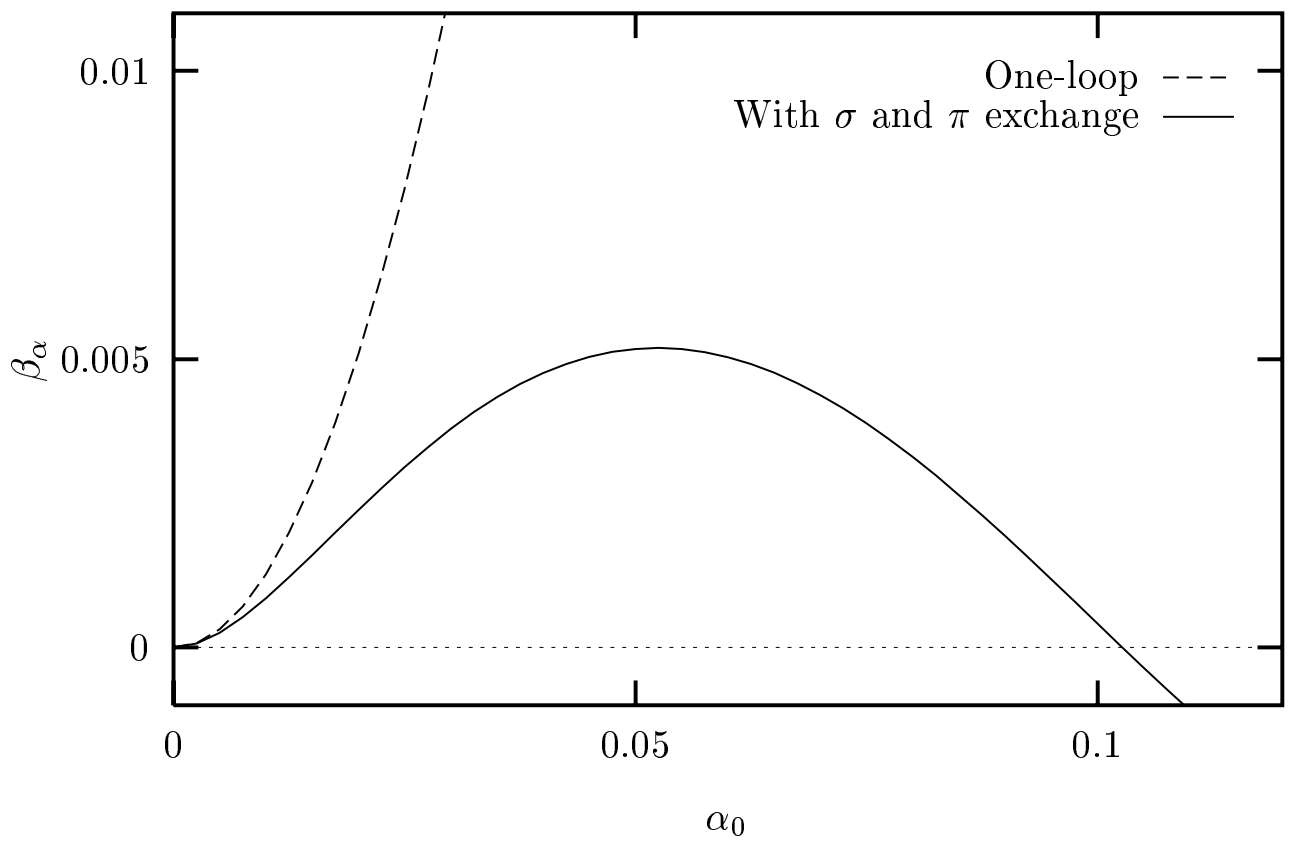

FIG. 10. The one-loop $\beta$ function for $N=60$ compared with the $\beta$ function including four-fermion interactions. 